\title{
Amyloid pathology disrupts gliotransmitter release in astrocytes
}

Anup G. Pillai ${ }^{1}$ and Suhita Nadkarni ${ }^{1 *}$

${ }^{1}$ Indian Institute of Science Education and Research Pune, Dr. Homi Bhabha Road, Pune, India

*Corresponding author, email address: suhita@iiserpune.ac.in 


\begin{abstract}
Accumulation of amyloid- $\beta$ peptide $(\mathrm{A} \beta)$, a hallmark of Alzheimer's disease (AD), is associated with synchronous hyperactivity and dysregulated $\mathrm{Ca}^{2+}$ signaling in hippocampal astrocytes. However, the consequences of altered $\mathrm{Ca}^{2+}$ signaling on the temporal dynamics of $\mathrm{Ca}^{2+}$ and gliotransmitter release events at astrocytic microdomains are not known. We have developed a detailed biophysical model of microdomain signaling at a single astrocytic process that accurately describes key temporal features of $\mathrm{Ca}^{2+}$ events and $\mathrm{Ca}^{2+}$-mediated kiss-and-run and full fusion exocytosis. Using this model, we ask how aberrant plasma-membrane $\mathrm{Ca}^{2+}$ pumps and mGluR activity, molecular hallmarks of $\mathrm{A} \beta$ toxicity that are also critically involved in $\mathrm{Ca}^{2+}$ signaling, modify astrocytic feedback at a tripartite synapse. We show that $\mathrm{AD}$ related molecular pathologies increase the rate and synchrony of $\mathrm{Ca}^{2+}$ and exocytotic events triggered by neuronal activity. Moreover, temporal precision between $\mathrm{Ca}^{2+}$ and release events, a mechanism indispensable for rapid modulation of synaptic transmission by astrocytes, is lost in AD astrocytic processes. Our results provide important evidence on the link between AD-related molecular pathology, dysregulated calcium signaling and gliotransmitter release at an astrocytic process.
\end{abstract}

Keywords: Astrocytes, calcium signaling, gliotransmitter release, synchrony, Alzheimer's disease 


\section{Introduction}

Bidirectional communication between neurons and astrocytes is crucial for normal brain function ${ }^{1-5}$. Despite the controversial role of astrocytes in information processing, it is well established that neuronal activity drives astrocytic $\mathrm{Ca}^{2+}$ signaling and concomitant release of gliotransmitters ${ }^{6-8}$. In parallel, disrupted astrocytic signaling has been implicated in several pathological conditions including cognitive decline and memory loss in $\mathrm{AD}^{9,10}$. Both in vivo and in vitro studies further confirm that accumulation of amyloid- $\beta$ (A $\beta)$, a pathological hallmark of $\mathrm{AD}$, disrupts astrocytic $\mathrm{Ca}^{2+}$ signaling and astrocytic glutamate release ${ }^{10-15}$. Indeed, elevated resting $\mathrm{Ca}^{2+}$ levels and enhanced spontaneous and synchronous $\mathrm{Ca}^{2+}$ events have been observed in vivo in $\mathrm{AD}$ astrocytes ${ }^{14}$. Despite this evidence, the causal relationship between amyloid pathology, astrocytic $\mathrm{Ca}^{2+}$ hyperactivity and its downstream effects on gliotransmitter release at a single process has not been examined.

$\mathrm{Ca}^{2+}$ excitability in astrocytes primarily depends on metabotropic glutamate receptor (mGluR)-mediated generation of inositol-3-phosphate $\left(\mathrm{IP}_{3}\right)$ and subsequent activation of $\mathrm{IP}_{3}$ receptors that release $\mathrm{Ca}^{2+}$ from the endoplasmic reticulum (ER $)^{7}$. Enhanced $\mathrm{mGluR}_{5}$ expression and high ER to cytosol volume ratio of peripheral astrocyte processes (PAPs) that surround majority of cortical synapses allow fast and highamplitude $\mathrm{Ca}^{2+}$ transients in these somewhat isolated glial compartments ${ }^{16-25}$. This specialized design together with presence of multiple $\mathrm{Ca}^{2+}$ sensors, vesicle types and recycling rates can trigger fast synchronous and slow asynchronous gliotransmitter release from individual astrocytic processes ${ }^{26,27}$. Measurements of individual exocytotic events indicate that mGluR stimulation leads to rapid kiss-and-run release of resident (docked) vesicles followed by slower full collapse fusions of newly recycled vesicles ${ }^{22}$. Consistent with this observation, several synaptotagmins (Syts) associated with exocytosis in neurons, including Syt4 and Syt7, have been reported in astrocytes ${ }^{28,29}$. Syt4 structurally resemble Syt 1 , the fast neuronal $\mathrm{Ca}^{2+}$ sensor, and regulate rapid kiss-and-run fusions in astrocytes ${ }^{29}$, while $S y t 7$, the neuronal asynchronous release sensor ${ }^{30}$, is largely associated with slow full fusion collapse of a vesicle ${ }^{31-33}$. 
Equipped with this elaborate machinery for exocytosis that is analogous to the presynaptic terminal in neurons, individual $\mathrm{Ca}^{2+}$ events in astrocytic microdomains exhibit precise spatiotemporal correlation with kiss-and-run release of a resident (docked) vesicle ${ }^{22}$. This correspondence between gliotransmitter release and calcium events is an integral part of synaptic transmission at a 'tripartite synapse' and critical for synchronization of neuronal activity ${ }^{8,34}$.

CA3 to CA1 synapses in the hippocampus are a potential neural substrate for various forms of learning and memory storage ${ }^{35}$. Encoding information in these synapses is input and synapse specific ${ }^{36,37}$. Anatomical and imaging studies show that astrocytic processes are in close proximity with most synapses in the hippocampus ${ }^{19,38,39}$. These findings together with their ability to generate calcium-induced gliotransmitter release suggest astrocytic processes as the critical center that facilitates information processing at individual synapses ${ }^{40}$.

While extensive experimental data and computational studies exists on somatic $\mathrm{Ca}^{2+}$ dynamics in astrocytes, a quantitative formulation of $\mathrm{Ca}^{2+}$ dynamics at a single astrocytic process and its relationship to $\mathrm{Ca}^{2+}$ mediated kiss-and-run and full fusion exocytosis has not been described ${ }^{41-44}$. We have developed a detailed biophysical model that incorporates multiple $\mathrm{Ca}^{2+}$ and $\mathrm{IP}_{3}$ signaling pathways alongside an elaborate kinetic scheme for synaptotagmins and vesicle recycling. The model reproduced $\mathrm{Ca}^{2+}$ and gliotransmitter release events at a single astrocytic process surrounding a typical CA3-CA1 synapse and is in quantitative agreement with experimentally observed $\mathrm{Ca}^{2+}$ and vesicular release events evoked by mGluR stimulation ${ }^{22}$. Using this modeling framework, we predict calcium activity and gliotransmitter releases at a single PAP in response to a range of presynaptic neurotransmitter release rates as well as characterize the consequences of AD-related molecular alterations in astrocytic signaling. Our results provide novel insights towards understanding how dysregulated astrocytic $\mathrm{Ca}^{2+}$ and gliotransmitter release can lead to aberrant synaptic signaling at a tripartite synapse in $\mathrm{AD}^{45}$. 


\section{Methods}

The calcium dynamics described by equations 1 and 2 combine fluxes $(J x)$ from various $\mathrm{Ca}^{2+}$ sources and $\mathrm{Ca}^{2+}$ regulating mechanisms that are present in the cytosol and ER, respectively (Fig. 1a). Equation 3 describes the production and degradation pathways of $\mathrm{IP}_{3}$, the intracellular signaling messenger. In the next sections we provide a detailed description of all $\mathrm{Ca}^{2+}$ and $\mathrm{IP}_{3}$ fluxes. Model parameters for all the components are in Tables 3-5.

$$
\begin{aligned}
& \frac{d}{d t} C a_{c y t}^{2+}=J_{P M C A}+J_{C y t B}-J_{S E R C A}+J_{\text {ER-leak }}+J_{I P 3 R}+J_{S y t} \\
& \frac{d}{d t} C a_{E R}^{2+}=\left[\left(J_{S E R C A}-J_{\text {ER-leak }}-J_{I P 3 R}\right)\left(\frac{V_{C y t}}{V_{E R}}\right)\right]+J_{E R B} \\
& \frac{d}{d t} I P_{3}=J_{m G l u R}-J_{I P 3 K}-J_{I P 3 P}+J_{P L C \delta}
\end{aligned}
$$

\section{Calcium dynamics}

The model includes multiple $\mathrm{Ca}^{2+}$ regulating components that are present on the plasma membrane, cytoplasm and ER. Plasma membrane high affinity $\mathrm{Ca}^{2+}$ ATPases (PMCAs) have been implicated in the maintenance of both resting and activity-dependent $\mathrm{Ca}^{2+}$ levels in astrocytes ${ }^{46}$. A three-state kinetic model captured the fast action of 2000 PMCAs, whereas slow action of cytosolic and ER $\mathrm{Ca}^{2+}$ buffers were modelled with two-state reaction equations (Tables $1 \& 2$ ). The parameters of cytosolic $\mathrm{Ca}^{2+}$ buffers (conc: $50 \mu \mathrm{M} ; \mathrm{K}_{\mathrm{D}}: 20 \mu \mathrm{M}$ ) were adjusted to reproduce the fast kinetics of mGluR-dependent $\mathrm{Ca}^{2+}$ transients in processes as reported by previous studies ${ }^{47}$. We assumed low endogenous $\mathrm{Ca}^{2+}$ buffering in individual astrocytic processes similar to what has been observed in dendritic spines that promotes fast and high amplitude transients ${ }^{48}$. Apart from PMCAs and $\mathrm{Ca}^{2+}$ buffers, sarcoplasmic/ER $\mathrm{Ca}^{2+}$ ATPases (SERCAs) also have a key role in maintaining resting $\mathrm{Ca}^{2+}$ levels in both cytosol and ER. A Hill equation 
(Table 1) captured the slow SERCA activity whose $\mathrm{Ca}^{2+}$ pumping rate was adjusted to match the ER lumen $\mathrm{Ca}^{2+}$ concentration $(100-800 \mu \mathrm{M})^{49}$. As a result, ER resting $\mathrm{Ca}^{2+}$ level $(\sim 400 \mu \mathrm{M})$ and pumping rate $\left(250 \mu \mathrm{M} \mathrm{s}^{-1}\right)$ in the model are higher compared to values in previous theoretical studies, $2-10 \mu \mathrm{M}$ and $\sim 1$ $\mu \mathrm{M} \mathrm{s}^{-1}$, respectively ${ }^{41,50,51}$. These considerations were important to reproduce the fast and high amplitude $\mathrm{Ca}^{2+}$ transients found at processes that primarily arise from stochastic opening of $\mathrm{IP}_{3}$-receptors on the ER membrane ${ }^{22,23}$. The ER membrane has a single cluster of $5 \mathrm{IP}_{3} \mathrm{R}$ channels to resemble the patchy staining of $I_{3} R_{2}$ on ER cisternae that generates the rapid $\mathrm{Ca}^{2+}$ puffs as reported by previous studies ${ }^{16,52}$. The model reliably captured the stochastic $\mathrm{Ca}^{2+}$ transients using a Langevin approximation of the Li-Rinzel $\mathrm{IP}_{3} \mathrm{R}$ model (Table 1) developed by Shuai et. $\mathrm{al}^{51}$. A passive $\mathrm{Ca}^{2+}$ leak channel maintained steady state ER $\mathrm{Ca}^{2+}$ level, although its precise role, despite its association with AD-related mutations, is not fully understood ${ }^{53}$.

\section{$I P_{3}$ dynamics}

While the major $\mathrm{IP}_{3}$ generation pathway is through the activation of mGluRs by glutamate spillover from adjacent presynaptic terminals, $\mathrm{IP}_{3}$ is also generated in a $\mathrm{Ca}^{2+}$-dependent manner through $\mathrm{PLC}_{\delta}$ activity. $\mathrm{IP}_{3}$ degradation in astrocytes are thought to be mediated by two separate molecular pathways, namely, inositol polyphosphate 5-phosphatase and $\mathrm{IP}_{3}$ 3-kinase, the former operates via a $\mathrm{Ca}^{2+}$-independent mechanism while the latter is $\mathrm{Ca}^{2+}$ activated ${ }^{54}$. The model approximated all these slow $\mathrm{IP}_{3}$ regulation mechanisms using four separate Hill equations (Table 2).

\section{Gliotransmitter release}

We modelled $\mathrm{Ca}^{2+}$-dependent gliotransmitter release using detailed kinetics schemes for two distinct synaptotagmins (Syt4 and Syt7) as well as the pathways of endocytosis, recycling and vesicle docking (Fig. 2a and supplementary Fig. 5). Syt4, has low $\mathrm{Ca}^{2+}$ affinity $\left(\mathrm{K}_{\mathrm{D}}: 22 \mu \mathrm{M}\right)$ and fast forward reaction rates compared to Syt7. Syt4 can therefore be activated in tight spaces close to the plasma membrane and ER 
where the vesicles are docked and $\mathrm{Ca}^{2+}$ transients are fast and have high amplitudes ${ }^{22}$. In comparison, Syt 7 has high $\mathrm{Ca}^{2+}$ binding affinity $(\sim 15 \mu \mathrm{M})$ but slow binding rates. Low amplitude and but long lasting $\mathrm{Ca}^{2+}$ rises can therefore activate $S y t 7$. As a consequence, $\mathrm{Ca}^{2+}$ events that are generated far from a vesicle can still contribute to the overall gliotransmitter release.

Consistent with the difference in $\mathrm{Ca}^{2+}$ binding kinetics of the two synaptotagmins, Syt4 and Syt7 coordinate distinct modes of release from different vesicle pools. Syt4 is associated with rapid kiss-andrun exocytosis of the docked vesicles close to the juxtaposition between ER and plasma membrane, whereas $S y t 7$ is associated with slow full fusion releases $28,29,33,55$. Interestingly, mobile vesicles that are 'not' localized in close spaces where $\mathrm{Ca}^{2+}$ concentration is high have been observed in astrocytic processes $^{56}$. These vesicles are likely to be newly recycled and not docked and their release can be attributed to full fusion via Sty7. In summary, high affinity binding sites in $S y t 7$ are activated by low amplitude $\mathrm{Ca}^{2+}$ elevations and promote slow full fusions of mobile vesicles, while docked vesicles are exocytosed in kiss-and-run mode by Syt4. Each of these pathways also follow distinct rates of endocytosis and recycling and have been modelled accordingly ${ }^{22}$.

We have systematically collated data from diverse experimental studies to refine the model parameters $^{22,24,57,58}$. Despite differences in experimental settings, temporal release histograms collected from these multiple studies on gliotransmitter release exhibited striking similarities in rise time, peak and decay time (Supplementary Fig. 2). From the reported values of imaging area $\left(\sim 1115 \mu \mathrm{m}^{2}\right)$ and TIRF evanescent field $(\sim 100 \mathrm{~nm})$ by these studies, we calculated an average imaging volume of roughly 111 $\mu \mathrm{m}^{3}$ per astrocyte. Together with previous measurements on total astrocytic volume $\left(24,465 \mu \mathrm{m}^{3}\right)$ and the number of processes $(100,000)$ in the CA1 layer of the rodent hippocampus, we estimated between 400 500 processes within the imaging volume ${ }^{20,59}$. Cumulative distributions of release histograms from these studies also indicated that a maximum of $\sim 400$ vesicles are released within this volume (Supplementary 
Fig. 3). We therefore, collected data from 400 independent simulation trials of a single astrocytic process to match the model results with experimental data. At resting state, $80 \%$ of the total vesicles are docked while the rest are in the mobile pool.

A typical chain of events starts with presynaptic release that increases extracellular glutamate concentration $(\sim 200 \mu \mathrm{M})$. We used a simple first order reaction equation (tau $=6.25 \mathrm{~ms}$ ) to capture the rapid rise and decay $(\sim 10 \mathrm{~ms})$ of glutamate levels near astrocytic processes ${ }^{60}$. Individual release events activated mGluRs to increase intracellular $\mathrm{IP}_{3}$ levels $(\sim 175 \mathrm{nM})$ that decayed to resting levels $(\sim 160$ $\mathrm{nM})$. The increase in $\mathrm{IP}_{3}$ caused cytoplasmic $\mathrm{Ca}^{2+}$ transients by stochastic opening of $\mathrm{IP}_{3} \mathrm{Rs}$ on the ER membrane (Supplementary Fig. 1). The change in $\mathrm{Ca}^{2+}$ level was small $(5-20 \mathrm{nM})$ at low frequency of activation. However, at higher rates of presynaptic glutamate release, the rapid build of $\mathrm{IP}_{3}$ generated high amplitude $\mathrm{Ca}^{2+}$ events $(5-10 \mu \mathrm{M})$ that activated synaptotagmins and caused concomitant gliotransmitter release events. We characterized the release machinery by clamping intracellular $\mathrm{Ca}^{2+}$ levels and measuring peak release (R) rate which was fitted to a Hill equation (4) with Vmax, Kd and coeff as free parameters.

$$
R\left(C a_{2+}\right)=V_{\max } \frac{\left(\mathrm{Ca}^{2+}\right)^{\text {coeff }}}{K_{d}^{\text {coeff }}+\left(\mathrm{Ca}^{2+}\right)^{\text {coeff }}}
$$

We additionally fitted events rates $(H)$ for $\mathrm{Ca}^{2+}$ and gliotransmitter release with a Hill equation (Eq. 5) to quantify its relationship with stimulus train frequency $(f)$. Where Hmin, Hmax and $\mathrm{EC}_{50}$ refer to minimum, maximum and half-max of event rate. All the parameters were fit recursively using the NelderMead simplex algorithm.

$$
H(f)=H_{\min }+\frac{\left(H_{\max }-H_{\min }\right)}{\left(1+\left(\frac{E C_{50}}{f}\right)\right)}
$$




\section{Data analysis}

\section{Calcium responsiveness measure}

We computed $\mathrm{Ca}^{2+}$ responsiveness for a single process by averaging $\mathrm{Ca}^{2+}$ signals across trials $(\mathrm{N}=1000)$. Astrocytic process was stimulated with the spillover glutamate profile from neuronal release at different firing frequencies $(0.1-100 \mathrm{~Hz})$ that lasted for 30 secs. The responsive measure $r$ was computed by normalizing the area under the curve as described in the below equation (Eq. 6), where $t_{0}$ and $T_{\text {stim }}$ refer to stimulation onset and duration, respectively.

$$
r=\int_{t_{0}}^{t_{0}+T_{\text {stim }}}\left[C a_{c y t}^{2+}\right](t) d t \div \int_{t_{0}-T_{\text {stim }}}^{t_{0}}\left[C a_{c y t}^{2+}\right](t) d t
$$

\section{Synchrony measure}

We computed synchrony of $\mathrm{Ca}^{2+}$ and release events from independent trials $(\mathrm{N}=1000)$ of a single astrocytic process stimulated with the same protocol as explained above. Event times for $\mathrm{Ca}^{2+}$ transients corresponded to the peak time of all the $\mathrm{Ca}^{2+}$ spikes whose amplitudes were above $300 \mathrm{nM}$. Individual release events were obtained from Poisson distribution of the instantaneous release rate at each simulation

time step $(50 \mu \mathrm{s})$. For each stimulation frequency, we calculated the Pinsky-Rinzel measure of synchrony ${ }^{61}$ from the set of phases, $l_{k}(j, m)$ for every $m^{\text {th }}$ event in $j$ th trial (Eq. 7). Where $\mathrm{k}, 1 \leq \mathrm{k} \leq N_{j}^{m}$, corresponds to all the events within the time interval $\left[\mathrm{T}_{j}^{m}, \mathrm{~T}_{j}^{m+1}\right)$ from all trials excluding the $j$ th trial itself. The operation transforms event times to a corresponding value spanning from 0 to 1 .

$$
\phi_{k}(j, m)=\frac{T_{k}(j, m)-T_{j}^{m}}{T_{j}^{m+1}-T_{j}^{m}}, T_{j}^{m} \leq T_{k}(j, m) \leq T_{j}^{m+1}
$$


Synchrony was then calculated from the vector of phases using the Strogatz and Mirollo ${ }^{62}$ method as defined below (Eq. 8)

$$
r(\phi(j, m))=\left(1 / N_{j, m}\right) \sum e^{\left(2 \pi i \phi_{k}(j, m)\right)}
$$

Overall synchrony for the set of $\mathrm{N}$ trials was obtained from the real part of $\mathrm{r}(\mathrm{l}(\mathrm{j}, \mathrm{m}))$ averaged across all the trials $(j)$ and events $(m)$. A value of 1 corresponds to a perfect alignment of event times across all the trials whereas 0 meant events arriving randomly.

\section{Computation of correlation between $\mathrm{Ca}^{2+}$ and gliotransmitter release events}

We quantified time-varying correlation between $\mathrm{Ca}^{2+}$ and gliotransmitter release events across independent trials using normalized joint peristimulus time histogram (nJPSTH), a method introduced by Aertsen at al. to compute correlations between neuronal spike trains ${ }^{63}$. For each trial of the stimulus protocol similar to the one described above for $\mathrm{Ca}^{2+}$ responsiveness, we computed peristimulus time histograms (PSTHs, $100 \mathrm{~ms}$ bins) for both $\mathrm{Ca}^{2+}$ and release events. For each non-zero bin of the PSTHs, the value of JPSTH matrix whose row and column indices correspond to the PSTH time bins of release and $\mathrm{Ca}^{2+}$ events, respectively, was incremented by one. The above procedure was repeated for all the 1000 trials. The JPSTH matrix was normalized by subtracting and dividing it with the matrix product and standard deviations, respectively, of normalized $\mathrm{Ca}^{2+}$ and release PSTHs. Cross-correlation was computed by summing the para-diagonal bins of the normalized JPSTH matrix from one second before to 11 seconds after the stimulus. Coincidence histogram was obtained from values along the main diagonal of the nJPSTH matrix. We quantified the relationship between peak cross-correlation across different stimulation frequencies by fitting to a logistic function as described below (Eq. 9). Where, $C_{\text {top }}$ and $C_{\text {slope }}$ 
refer to peak and steepness of the response, while $f_{\text {mid }}$ is the frequency at which response attains halfmaximum.

$$
C(f)=\frac{C_{\text {top }}}{1+C_{\text {slope }} e^{-\left(f \cdot f_{\text {mid }}\right)}}
$$

\section{Numerical simulation}

The simulations were run with $50 \mu$ s time step on the institution's HPC facility (1500 nodes, PBSPro scheduler). The data was analyzed using custom Python scripts (www.python.org).

\section{Results}

\section{Model of $\mathrm{Ca}^{2+}$ signaling at a single astrocytic process}

A schematic of the $\mathrm{Ca}^{2+}$ model is illustrated in figure 1a. The model has two coupled compartments for $\mathrm{Ca}^{2+}$ dynamics, (1) an intracellular cytoplasmic space and (2) ER compartment. PMCA pumps on the plasma membrane mediate slow bidirectional $\mathrm{Ca}^{2+}$ flow, whereas rapid $\mathrm{Ca}^{2+}$ elevations within a process primarily arise from stochastic opening of $\mathrm{IP}_{3}$ receptors present on the ER membrane. The kinetics of $\mathrm{Ca}^{2+}$ spikes are further shaped by $\mathrm{Ca}^{2+}$ buffers and SERCAs. The model captures stochastic $\mathrm{Ca}^{2+}$ dynamics in single astrocytic processes upon mGluR stimulation (details of each component are in the Methods section).

The study by Marchaland et al. reported on the characteristics of $\mathrm{Ca}^{2+}$ events from single astrocytic processes activated by DHPG $(100 \mu \mathrm{M}, 2$ secs $)$, a highly specific group 1 mGluR agonist ${ }^{22}$. Using the same experimental paradigm, we first examined $\mathrm{Ca}^{2+}$ event characteristics from 400 independent runs of the single process model. Representative time courses and overlay of stochastic $\mathrm{Ca}^{2+}$ spikes from a subset 
of trials are presented in figures $1 \mathrm{~b}$ and c. Peak amplitude, rise time and decay constant of the events were normally distributed with peaks at $2.4 \mu \mathrm{M}, 80 \mathrm{~ms}$ and $120 \mathrm{~ms}$, respectively (Figs. 1d \& e). In good agreement with the experimental results, we find that the temporal distribution of $\mathrm{Ca}^{2+}$ events decayed exponentially ( $\tau=378 \mathrm{~ms}$ ) (Fig. 1f). The model's close match with experimental result is further evident from the close overlap ( $\sim 90 \%)$ between $\mathrm{Ca}^{2+}$ event cumulative histograms (Fig. 1f, inset).

\section{Modeling vesicular release at a single astrocytic process}

Despite several reports on $\mathrm{Ca}^{2+}$-dependent gliotransmitter release from astrocytes, a biophysical framework that links calcium rise at a single process to kiss-and-run and full fusion exocytosis is not present. Based on a broad understanding of astrocytic $\mathrm{Ca}^{2+}$-sensors (synaptotagmins), vesicle distribution and recycling times, we propose a detailed kinetic scheme for the astrocytic release machinery at a single process (Fig. 2a \& Supplementary Fig. 5).

Two synaptotagmins, Syt4 and Syt7, have been reported in astrocytes ${ }^{28,29,33,64}$. Syt 4 has a single low affinity domain $\left(\mathrm{C}_{2} \mathrm{~B}\right)$ that binds $2 \mathrm{Ca}^{2+}$ ions with fast forward rates ${ }^{31}$. In contrast, Syt7 has two calcium domains $\left(\mathrm{C}_{2} \mathrm{~A}\right.$ and $\left.\mathrm{C}_{2} \mathrm{~B}\right)$ that have high affinity but slow forward reaction rates ${ }^{65}$. This makes Syt7 a slow calcium sensor that operates under low $\mathrm{Ca}^{2+}$ levels. TIRF imaging studies using fluorescently labelled vesicles also indicate fast kiss-and-run-like confined releases alongside slow spreading full fusions events ${ }^{27,32}$. It is also established that Syt4 with a single $\mathrm{Ca}^{2+}$ binding domain can only promote kiss-and-run exocytosis ${ }^{31}$. Conversely, capacitance measurements in astrocytes that primarily reflects full fusion exocytosis estimated a Hill's coefficient of 5 that match with the $\mathrm{Ca}^{2+}$ binding sites in $\mathrm{Syt} 7^{66}$.

Based on these findings, we modeled two separate $\mathrm{Ca}^{2+}$ sensors, Syt4 and Syt7, that have different $\mathrm{Ca}^{2+}$ affinities and independently mediate fast (kiss-and-run release) and slow (full fusion) gliotransmitter release respectively, in astrocytic process. Syt4, having low $\mathrm{Ca}^{2+}$ affinity and fast reaction kinetics, is 
primarily activated by sharp and high amplitude $\mathrm{Ca}^{2+}$ events that have been observed near ER tubuli. On the other hand, $\mathrm{Syt} 7$, with high $\mathrm{Ca}^{2+}$ affinity and slow kinetics, specifically respond to slow and low amplitude $\mathrm{Ca}^{2+}$ rises. Analogous to vesicle recycling at the presynaptic terminal ${ }^{67}$, we further assumed that every vesicle once released is subsequently endocytosed and transported (mobile) to a docking site (Fig. 2a). Separately, it has also been suggested that kiss-and-run and full fusion may arise from discrete vesicle pools ${ }^{22,27}$. Estimates on release parameters including release rate, vesicles per process and rates of endocytosis and vesicle recycling were obtained from published literature and are detailed in Tables 1-5.

Similar to what has been studied at the presynaptic terminal, we first examined the relationship between $\mathrm{Ca}^{2+}$ and peak release rate at a single process ${ }^{68,69}$. Clamping intracellular $\mathrm{Ca}^{2+}$ transiently increased release rate that decayed back to zero in tens of milliseconds (Fig. 2b). Estimates on the release machinery parameters, $K_{D}$ and binding sites (slope), were obtained by fitting the peak release rate to a Hill equation (Eq. 4; Fig. 2c). Decay of time-to-peak release was fit with a bi-exponential function with two time constants (Fig. 2d).

We next examined gliotransmitter release from 400 independent processes to validate the model results with previous experimental studies that measured single exocytotic events from entire astrocytes $22,24,57,58$. Similar to the study by Marchaland et al., we stimulated astrocytic process with DHPG (100 $\mu \mathrm{M}, 2$ secs) to evoke $\mathrm{Ca}^{2+}$ transients and concomitant gliotransmitter events ${ }^{22}$. A representative raster plot of kiss-andrun and full fusion events from a subset of astrocytic process stimulated with DHPG is shown in figure 2e. The model indicates rapid rise in release events that peaked at roughly $250 \mathrm{~ms}$ after the stimulus onset and decayed exponentially (Fig. 2f). Both temporal and cumulative release histograms (Fig. 2f inset) from the model are in good agreement with the study by Marchaland et al. The model further indicates that a majority of the vesicles are exocytosed immediately after the stimulus via kiss-and-run mode followed by slow and asynchronous full fusions (Fig. 2g). Total number of docked vesicles from all the processes 
decreased rapidly after DHPG application while the number of vesicles in the mobile pool increased. Similar to what has been observed experimentally, the release, endocytosis and reacidification of docked vesicles are faster compared to mobile vesicles (Figs. 2h-i)

\section{Modeling astrocytic processes with AD pathology}

mGluR signaling and PMCA pump activity, two critical regulators of astrocytic calcium signaling, are modified in AD. Specifically, mGluRs responses are enhanced in $\mathrm{AD}$ astrocytes ${ }^{13,70,71}$, while $\mathrm{A} \beta$ inhibits $\mathrm{Ca}^{2+}$ signaling through PMCAs ${ }^{72-74}$.

Similar what has been observed experimentally, we up-regulated mGluR signaling in the AD-mGluR group by increasing its affinity for glutamate $\left(\mathrm{K}_{\mathrm{D}}\right.$, control: $6 \mu \mathrm{M}$, AD-mGluR: $\left.3 \mu \mathrm{M}\right)$ and peak $\mathrm{IP}_{3}$ production rate $\left(V_{\max }\right.$, control: $\left.0.65 \mu \mathrm{Ms}^{-1}, \mathrm{AD}-\mathrm{mGluR}: 1.4 \mu \mathrm{Ms}^{-1}\right)$. As expected, peak $\mathrm{IP}_{3}$ response to constant glutamate stimulation lasting $30 \mathrm{sec}$ is both higher and left shifted in the AD-mGluR group in comparison to the control group, indicating an overall increase in $\mathrm{IP}_{3}$ production (Fig. 3a).

We next modeled the shift PMCA's $\mathrm{Ca}^{2+}$ dependency in the AD-PMCA group by decreasing its affinity for $\mathrm{Ca}^{2+}\left(\mathrm{K}_{\mathrm{D}}\right.$, control: $1.3 \mu \mathrm{M}$, AD-PMCA: $\left.4 \mu \mathrm{M}\right)$. In the AD-PMCA group, the decrease in PMCA Ca ${ }^{2+}$ affinity could be observed as a rightward shift in its steady-state outward $\mathrm{Ca}^{2+}$ flux when the level of intracellular $\mathrm{Ca}^{2+}$ was clamped to different concentrations (Fig. 3b). An interesting side effect of decreased PMCA efficiency is the rise in resting $\mathrm{Ca}^{2+}$ levels in both the ER and cytoplasm in the ADPMCA astrocytic process (control: $80 \mathrm{nM}$, AD-PMCA: $166 \mathrm{nM}$ ) (Figs. 3c \& e). However, resting $\mathrm{IP}_{3}$ levels where not affected in any of the AD groups (Fig. 3d).

We further probed the effect of PMCA and mGluR manipulation on astrocytic $\mathrm{Ca}^{2+}$ dynamics by stimulating control and all AD groups with a standard stimulus protocol used in several experimental 
studies that examined mGluR-mediated $\mathrm{Ca}^{2+}$ signaling in astrocytes ${ }^{75,76}$. Consistent with experimental results, prolonged glutamate application $(6 \mu \mathrm{M}, 120 \mathrm{~s})$ induced $\mathrm{Ca}^{2+}$ spikes in a majority $\mathrm{AD}$ astrocytic process (Figs. 3f-i, colors represent independent trials). $\mathrm{Ca}^{2+}$ oscillations were quantified using the responsiveness measure which is a readout for cumulative response for the entire stimulus duration (See Model and Methods for details). All the AD astrocytic groups displayed higher responsiveness and $\mathrm{Ca}^{2+}$ event rate compared to control, this is evident from the leftward-shift in the dose-response curves (Fig s. 3j-k). Interestingly, AD groups with impaired PMCA signaling exhibited spontaneous $\mathrm{Ca}^{2+}$ events even at a very low glutamate concentration $(<1 \mu \mathrm{M})$ where the control astrocytic process is silent (Fig. 3k).

$\mathrm{Ca}^{2+}$ events are enhanced in astrocytic process with AD pathology

Several studies have reported increased $\mathrm{Ca}^{2+}$ activity in astrocytes from $\mathrm{AD}$ animal models and when treated with amyloid- $\beta^{11,77}$. Additionally, astrocytes with AD pathology also exhibit unusually high spontaneous $\mathrm{Ca}^{2+}$ activity ${ }^{14,77,78}$. Exploring further on our previous results on astrocytic responses to constant glutamate pulses, we next quantified $\mathrm{Ca}^{2+}$ signaling in a $\mathrm{n}$ astrocytic process using a physiologically realistic stimulus with the same concentration and temporal profile of spillover glutamate from presynaptic release ${ }^{60}$ (details in materials and methods).

As representative examples we show $\mathrm{Ca}^{2+}$ transients evoked by spillover glutamate released at $3 \mathrm{~Hz}$ (Fig. 4a-d). $\mathrm{Ca}^{2+}$ events were more frequent and larger in amplitude in astrocytic process from all the AD groups compared to the control group. Additionally, in AD groups with impaired PMCAs, spontaneous events could be observed before the stimulus onset (Dotted box in Figs. 4c \& d).

To further characterize these $\mathrm{Ca}^{2+}$ responses, we stimulated astrocytic processes at different presynaptic release rates $(0.1-100 \mathrm{~Hz})$ resembling the broad range of neuronal activity observed in vivo (Figs. 4e-h). Half maximums of $\mathrm{Ca}^{2+}$ event rate $\left(\mathrm{EC}_{50}\right)$ obtained from fits to a Hill equation (Eq. 5) were low in all the 
$\mathrm{AD}$ groups compared to control astrocytic process (Fig. 4e), implying enhanced $\mathrm{Ca}^{2+}$ activity. Impairment in PMCA activity, apart from increasing spontaneous activity, also lowered rise time, full-width at halfmaximum and decay time in all the stimulation regimes (Figs. 4f-h).

\section{Gliotransmitter release events are enhanced in astrocytic process with AD pathology}

We next examined the impact of $\mathrm{AD}$-induced alterations on $\mathrm{Ca}^{2+}$-mediated exocytosis. A single astrocytic process was stimulated ( 1000 trials) with the temporal profile of spillover glutamate released at different rates $(0.1-100 \mathrm{~Hz})$. Representative raster plots $(30$ trials; $30 \mathrm{sec})$ of kiss-and-run and full fusion release from $3 \mathrm{~Hz}$ stimulation are described in figure 5 a-d.

Both kiss-and-run and full fusion exocytosis were upregulated in the AD-mGluR group compared to control. Accordingly, $\mathrm{EC}_{50}$ values obtained by fitting a Hill equation (Eq. 5) to kiss-and-run and full fusion rates were low in the AD-mGluR group compared to control (Figs. 5e \& f). The drastic reduction in kiss-and-run exocytosis was very evident in the $\mathrm{AD}$-mGluR group whose $\mathrm{EC}_{50}$ value was reduced to less than a third of the control group (Fig. 5e). In contrast, in AD astrocyte process groups with impaired PMCA (AD-PMCA \& AD-mGluR+PMCA), the rate of kiss-and-run exocytosis became unresponsive to changes in presynaptic glutamate release rate (Fig. 5e). Both the AD-PMCA groups also had high spontaneous release rates that were mostly kiss-and-run. In the AD-mGluR group, kiss-and-run release dominated at all stimulation frequencies. Whereas in AD groups with inhibited PMCA action, a majority of releases at high stimulation rates $(>10 \mathrm{~Hz})$ were full fusions. AD related abnormalities shifted the relationship between total release rate and stimulation rate leftward with low $\mathrm{EC}_{50}$ values in all the $\mathrm{AD}$ groups (In Hz, control: 10.2, AD-mGluR: 4.8, AD-PMCA: 3.7, AD-mGluR+PMCA: 1.5) (Fig. 5g).

Synchrony of $\mathrm{Ca}^{2+}$ and release events are enhanced in AD astrocytic processes with altered $m G l u R$ pathology 
Increased astrocytic synchronization in the form of coherent $\mathrm{Ca}^{2+}$ and gliotransmitter release events boosts neural synchrony in vivo and ex vivo ${ }^{79,80}$. Synchronous $\mathrm{Ca}^{2+}$ activity in astrocytes is also associated with pathological neuronal discharges in several disease models including epilepsy and $\mathrm{AD}^{14,81}$. We therefore tested synchrony of stochastic $\mathrm{Ca}^{2+}$ and release events from $\mathrm{AD}$ astrocytic process stimulated with glutamate pulses resembling neuronal release at different rates $(0.1-100 \mathrm{~Hz})$. Event synchrony between independent stimulation trials (1000) was computed using the Pinsky-Rinzel algorithm (See Materials and Methods for details).

A polar representation of phase coherence histogram computed for $0.2 \mathrm{~Hz}$ stimulation indicates high coherence of $\mathrm{Ca}^{2+}$ events in the AD group with enhanced mGluR signaling (Fig. 6a). However, when stimulated with different rates of presynaptic glutamate release, coherence of $\mathrm{Ca}^{2+}$ events was high in all AD groups at low rates $(<1 \mathrm{~Hz})$. In contrast, synchrony remained low at all frequencies in the control group (Fig. 6b). These findings on synchronous $\mathrm{Ca}^{2+}$ events at a single astrocytic processes is in agreement with previous whole-cell measurements in AD astrocytes in vivo (kuchibhotla2008). The model thus revels mGluR signaling, and not PMCA activity, as a cellular substrate for synchronous astrocytic hyperactivity.

Simultaneous measurements of $\mathrm{Ca}^{2+}$ and gliotransmitter release events from single astrocytic processes in response to physiological neuronal release is difficult and none exist for AD disease model. Using our systematically validated model we next describe synchrony of gliotransmitter releases in response to astrocytic $\mathrm{Ca}^{2+}$ activity (Fig. 6c and d). Similar to what we observed for $\mathrm{Ca}^{2+}$ events, coherence of gliotransmitter releases was high in the AD-mGluR group at $0.2 \mathrm{~Hz}$ (Fig. 6c). Although, gliotransmitter releases were much less synchronous than $\mathrm{Ca}^{2+}$ events in the same trials, at low stimulation rates, synchrony of AD-mGluR group dominated over all the other groups (Fig. 6d). 
Low probability of docked vesicles leads to loss in the temporal correlation between $\mathrm{Ca}^{2+}$ and release events in $A D$ astrocytic processes

Apart from synchronizing neuronal ensembles, astrocyte processes also exert synapse-specific control of synaptic transmission ${ }^{45}$. An important prerequisite for this functionality is precise temporal association between $\mathrm{Ca}^{2+}$ event arising out of presynaptic release and gliotransmitter release events at a specific tripartite synapse. The close association between calcium activity is clearly seen in experimental data and is reproduced accurately in our model (Figs. 1f and 2f). Following up on our previous results on increased calcium signaling and gliotransmission by $\mathrm{AD}$ mechanisms, we next investigated its impact on the temporal coincidence between $\mathrm{Ca}^{2+}$ and release events.

Similar to the experimental protocol described previously, we stimulated control and AD astrocytic process with the temporal profile of spillover glutamate at different release rates. Using joint crosscorrelation analysis (JCC), the temporal association between $\mathrm{Ca}^{2+}$ and gliotransmitter release events was computed for each stimulation regime (See Model and Methods for details). We first present data from correlation analysis and the nJPSTH matrix computed from 1000 trials of $10 \mathrm{~Hz}$ stimulation train for control and AD groups (Fig. 7a-d).

As evident from the colored representation of the nJPSTH matrix, in the control group, $\mathrm{Ca}^{2+}$ and gliotransmitter release events were highly coincident for more than $10 \mathrm{~s}$ from the stimulus onset (Fig. $7 \mathrm{a}$, left). This was further confirmed from the coincidence histogram computed from the diagonal elements of the nJPSTH matrix (Fig. 7a, top-right). In agreement with experimental findings ${ }^{22}$, cross-correlations obtained from para-diagonal bins of nJPSTH matrix peaked at $100 \mathrm{~ms}$ lag (Fig. 7a, bottom-right). 
However, compared to the control group, nJPSTH matrices of AD groups indicated lower coincidence between $\mathrm{Ca}^{2+}$ and gliotransmitter release events (Fig. 7a-d, top-left). Evidently, in all the AD groups, coincidence was low and decayed sharply after the stimulus onset (Fig. 7a-d, top-right). All the AD groups also had low and broadened cross-correlations values (Fig. 7a-d, bottom-right).

We next extended the cross-correlation analysis at a single astrocytic process to low and high stimulation frequencies $(0.4-100 \mathrm{~Hz})$. Similar to what we found with $\mathrm{Ca}^{2+}$ synchrony, peak cross-correlation was high in all the groups when the frequency stimulation was low. However, in stark contrast, $\mathrm{Ca}^{2+}$ and vesicular release events were less correlated in all the AD groups. Fits to a logistic function (Eq. 9) also yielded lower values in all the $\mathrm{AD}$ groups compared to the control group (Fig. 7e).

To further understand the reason behind this surprising result, we quantified average number of vesicles docked during stimulus presentation. Clearly, docked vesicles decreased with higher stimulation rates and was low in all the AD groups (Fig. 7f). Since docked vesicles mediate fast and synchronous kiss-and-run exocytosis, we next examined its link with peak cross-correlation between $\mathrm{Ca}^{2+}$ and gliotransmitter release events when stimulated by glutamate pulses delivered at the theta frequency range $(2-10 \mathrm{~Hz})($ Fig. $7 \mathrm{~g}$ ). The clear linear relationship between the two further imply rapid depletion of docked vesicles as the underlying mechanism for the loss in temporal coincidence between $\mathrm{Ca}^{2+}$ and release events in $\mathrm{AD}$ astrocytes.

\section{Discussion}

While AD clearly manifests as a synaptic abnormality early on, little is known about the contribution by astrocytes to its pathogenesis ${ }^{10,82}$. To address this gap, we harnessed experimental data across multiplescales and developed a biophysical model for astrocytic calcium response and ensuing gliotransmitter release at a typical CA3-CA1 synapse. The model is strongly rooted in extant experimental evidence and 
comprises of a realistic description of molecular components such as membrane receptors, local ER extent, vesicle types and importantly, the synaptotagmins. The model accurately captures statistical distributions, timescale and amplitudes of both $\mathrm{Ca}^{2+}$ and gliotransmitter release events as reported experimentally ${ }^{22}$. W e next characterized both $\mathrm{Ca}^{2+}$ signaling and vesicle release at a single process to show that both synchrony and rate of astrocytic $\mathrm{Ca}^{2+}$ responses to trains of presynaptic glutamate release are enhanced in AD. The most crucial insight that arises out of this study is the observed loss in temporal precision of individual $\mathrm{Ca}^{2+}$ and vesicle release events in all the $\mathrm{AD}$ astrocytic groups. The model predicts impaired mGluR and PMCA signaling to distinctly enhance $\mathrm{Ca}^{2+}$ synchrony and spontaneous hyperactivity, respectively. Together, our results provide novel mechanistic insights on AD-related astrocytic dysfunction as well as suggest impaired astrocytic feedback at tripartite synapses as a critical functional consequence of $\mathrm{AD}$ pathology.

A wealth of whole-cell $\mathrm{Ca}^{2+}$ imaging and computational studies have linked $\mathrm{IP}_{3} \mathrm{R}$-mediated slow calcium waves in astrocytes and long-range synchronization of neuronal networks $s^{34,80,83,84}$. The traditional view that astrocytes primarily impart slow modulation has been challenged recently by studies that reported high amplitude $\mathrm{Ca}^{2+}$ transients and $\mathrm{Ca}^{2+}$-mediated release from astrocytic processes ${ }^{22,85-87}$. Despite the indication that astrocytic processes can rapidly modulate synaptic transmission, there is no clear understanding of the biophysical mechanisms that mediate the tight temporal correspondence between $\mathrm{Ca}^{2+}$ events and vesicular release at a single process ${ }^{22,45,88,89}$. From the model it is clear that increased presence of ER, the high $\mathrm{Ca}^{2+}$ levels in PAPs, rapid opening of $\mathrm{IP}_{3} \mathrm{Rs}$, low cytoplasmic buffering, a presence of a fast $\mathrm{Ca}^{2+}$ sensor and rapid vesicle recycling together orchestrate the tight temporal feedback at tripartite synapses.

Based on experimental evidence on $\mathrm{Ca}^{2+}$ compartmentalization, we treated each astrocytic process as an independent unit with high (50\%) ER-to-cytosol volume ratio ${ }^{90}$. Calcium responses at these tiny domains 
are further constrained to tight subcellular domains due to diffusion constraints that increase the influence of $\mathrm{mGluR}_{5}$ and limits the spatial spread of $\mathrm{IP}_{3}$ within individual processes ${ }^{21,91}$. The presence of multiple $\mathrm{Ca}^{2+}$ regulating mechanisms and a small cluster of $\mathrm{IP}_{3} \mathrm{Rs}$ leads to stochastic $\mathrm{Ca}^{2+}$ events in astrocytic processes similar to $\mathrm{Ca}^{2+}$ puffs observed in other cell types ${ }^{70,92}$. Our stochastic model with a single cluster of five $\mathrm{IP}_{3} \mathrm{Rs}$ accurately reproduced both temporal characteristics and the distribution of $\mathrm{Ca}^{2+}$ event amplitudes, rise times and decay constants as reported by previous studies ${ }^{22-25}$. The model also reproduced several critical determinants of mGluR-mediated $\mathrm{Ca}^{2+}$ events, such as mGluRs dose-response, ER $\mathrm{Ca}^{2+}$ levels $(400 \mathrm{mM})$ and SERCA refilling time $(100 \mathrm{~s})^{49,93-96}$. Additionally, single process $\mathrm{Ca}^{2+}$ responsiveness to glutamate pulses from the model is also in good agreement with somatic $\mathrm{Ca}^{2+}$ imaging data recorded from astrocytic cultures ${ }^{4}$. Lastly, we compared model results to the temporal histogram of $\mathrm{Ca}^{2+}$ events from entire astrocytes ${ }^{22}$. Based on our estimate on the number of processes within the imaging volume of a single astrocyte, we pooled data from 400 independent simulation trials. While it is likely that there is variability in intrinsic parameters ( $\mathrm{IP}_{3}$ receptors, geometry etc) across astrocytic processes, our simulations account for more than $90 \%$ of all the events observed experimentally. These independent validations across a wide range of experimental data indicate that the biophysical model developed here is an accurate representation of $\mathrm{Ca}^{2+}$ signaling in astrocytic processes.

An important functional consequence of astrocytic $\mathrm{Ca}^{2+}$ events is gliotransmitter release. Indeed, both kiss-and-run and full fusions exocytosis with distinct timescales of exocytosis, endocytosis and reacidification have been reported in astrocytic processes ${ }^{22,27}$. This evidence supports two separate molecular pathways for vesicular release and recycling. Alongside two synaptotagmins (Syt4 \& Syt7) that differ in reaction kinetics and $\mathrm{Ca}^{2+}$ sensitivity, astrocytic processes are also thought to harbor docked and mobile vesicles $22,28,29,55,56,97$. Activation of Syt4 by fast and high amplitude $\mathrm{Ca}^{2+}$ events near the ER tubuli which is tightly juxtaposed to the plasma membrane near docked vesicles is thought to result in rapid kiss-and-run release ${ }^{22}$. On the other hand, Syt7 whose $\mathrm{Ca}^{2+}$ affinity is high can be triggered by low amplitude and slow $\mathrm{Ca}^{2+}$ events to result in slow asynchronous release of mobile vesicles. Indeed, EM 
studies also report that at astrocytic processes, vesicles could be seen as far as $200 \mathrm{~nm}$ away from the plasma membrane and a fraction of them are suggested to be mobile ${ }^{5,56}$. Considering the very limited spatial extent of a single process where only a few vesicles are docked, the presence of mobile vesicles provides an alternate pathway for experience dependent modulation of release kinetics. Notably, the proportion of slow full-fusion and fast transient releases in astrocytes is stimulus dependent ${ }^{27,32}$. In agreement to the above, we observed a shift from kiss-and-run to full fusion exocytosis in the AD astrocytic process.

In order to model $\mathrm{AD}$ astrocytes, we considered two well known molecular signatures of $\mathrm{A} \beta$ accumulation that are critical for astrocytic $\mathrm{Ca}^{2+}$ regulation, namely, dysfunctional PMCAs and elevated mGluR signaling ${ }^{13,70-73}$. Our study provides several clear and experimentally testable predictions that relate $A \beta$-induced alterations with astrocytic $\mathrm{Ca}^{2+}$ signaling at a single process level. Firstly, our prediction that $\mathrm{AD}$ astrocytic process have spontaneous $\mathrm{Ca}^{2+}$ activity is consistent with experimental reports from $\mathrm{AD}$ animals and ex vivo $\mathrm{A} \beta$ treatment $^{11,14,78,98}$. Our study specifically associates reduced PMCA activity with increased cytosolic resting $\mathrm{Ca}^{2+}$ and enhanced spontaneous $\mathrm{Ca}^{2+}$ activity. Again, increased resting intracellular $\mathrm{Ca}^{2+}$ has been reported in vivo in $\mathrm{AD}$ astrocytes, although the mechanism was not known ${ }^{14}$. Secondly, results from the model indicate enhanced mGluR signaling as the underlying molecular mechanism for $\mathrm{Ca}^{2+}$ event synchrony and similar to this finding, synchronous bulk $\mathrm{Ca}^{2+}$ signals are found in $\mathrm{AD}$ astrocytes ${ }^{14}$.

Despite the well-established evidence for abnormal $\mathrm{Ca}^{2+}$ signaling in astrocytes, there is no clear understanding of the consequences of abnormal calcium signaling on gliotransmitter release at a single process. We show that kiss-and-run and full fusion exocytosis mediated by Syt4 and Syt7, respectively, were differently affected by mGluR and PMCA mechanisms. Enhanced mGluR signaling increased both kiss-and-run and full fusion releases as well as their inter-trail synchrony when stimulated with trains of 
glutamate pulses mimicking presynaptic activity. In contrast, PMCA alteration abolished both synchrony and the logistic relationship of kiss-and-run release with stimulation strength.

Lastly, our study uncovered a very unique dependence between AD-related enhanced $\mathrm{Ca}^{2+}$ dynamics and loss in temporal precision of gliotransmitter release at a single process. Unlike in control astrocytes whose release events were concurrent with $\mathrm{Ca}^{2+}$ events within a time window of $100 \mathrm{~ms}$, all the $\mathrm{AD}$ groups displayed a loss in this precision very early on after the stimulus onset. We also report a clear linear relationship between this loss in temporal coincidence and rapid depletion of docked vesicles when stimulated in the theta frequency range $(2-10 \mathrm{~Hz})$ which also corresponds to the dynamic range of astrocytic responses at a single process. Thus when affected by AD pathology, astrocytic processes become more synchronous but lose their ability to provide timely feedback at single tripartite synapses ${ }^{99}$. As suggested by previous computational and experimental studies, both these mechanisms can affect how astrocytes modulate presynaptic release probability and synaptic information transfer in the hippocampus $^{1,89}$.

In summary, we incorporated diverse experimental data to construct a biophysically detailed model for calcium signaling and the corresponding gliotransmitter release at a single astrocytic process. The model accurately reproduces both the kinetics and statistical profiles of both calcium and release events as reported by previous experiments. The biophysical model we describe is firmly grounded in physiology and is flexible to simulate diverse experimental setting to predict novel astrocytic mechanisms on synaptic signaling. The difficulty in performing actual experiments at the highly ramified astrocytic processes further enhance the value of the model. Based on our model we show that in AD astrocytes, enhanced depletion of docked vesicles leads loss in correspondence between calcium events and gliotransmitter release events. We hypothesize that this mismatch in information transfer at a crucial hippocampal tripartite synapse may contribute to higher level cognitive deficits associated with AD. 


\section{Data availability}

All the codes of our model and simulations will be made publicly available at GitHub repository (https://github.com/anupgp/astrocytic_process) after publication.

\section{References}

1. Perea, G. \& Araque, A. Astrocytes potentiate transmitter release at single hippocampal synapses. Science (80-. ). (2007). doi:10.1126/science.1144640

2. Pougnet, J. T. et al. ATP P2X receptors downregulate AMPA receptor trafficking and postsynaptic efficacy in hippocampal neurons. Neuron 83, 417-430 (2014).

3. Andersson, M. \& Hanse, E. Astrocytes Impose Postburst Depression of Release Probability at Hippocampal Glutamate Synapses. J. Neurosci. 30, 5776-5780 (2010).

4. Wallach, G. et al. Glutamate Mediated Astrocytic Filtering of Neuronal Activity. PLoS Comput. Biol. 10, (2014).

5. Jourdain, P. et al. Glutamate exocytosis from astrocytes controls synaptic strength. Nat. Neurosci. 10, 331-339 (2007).

6. Agulhon, C. et al. What Is the Role of Astrocyte Calcium in Neurophysiology? Neuron 59, 932 $946(2008)$.

7. Bazargani, N. \& Attwell, D. Astrocyte calcium signaling: The third wave. Nat. Neurosci. 19, 182189 (2016).

8. Volterra, A., Liaudet, N. \& Savtchouk, I. Astrocyte Ca2+ signalling: An unexpected complexity. Nat. Rev. Neurosci. 15, 327-335 (2014).

9. Seifert, G., Schilling, K. \& Steinhäuser, C. Astrocyte dysfunction in neurological disorders: A molecular perspective. Nat. Rev. Neurosci. 7, 194-206 (2006).

10. Vincent, A. J., Gasperini, R., Foa, L. \& Small, D. H. Astrocytes in Alzheimer's disease: Emerging roles in calcium dysregulation and synaptic plasticity. J. Alzheimer's Dis. 22, 699-714 (2010).

11. Abramov, A. Y., Canevari, L. \& Duchen, M. R. Changes in intracellular calcium and glutathione in astrocytes as the primary mechanism of amyloid neurotoxicity. J. Neurosci. 23, 5088-95 (2003).

12. Abramov, A. Y., Canevari, L. \& Duchen, M. R. Calcium signals induced by amyloid $\beta$ peptide and their consequences in neurons and astrocytes in culture. Biochim. Biophys. Acta - Mol. Cell Res. 1742, 81-87 (2004). 
13. Grolla, A. A. et al. Amyloid-b and Alzheimer's disease type pathology differentially affects the calcium signalling toolkit in astrocytes from different brain regions. Cell Death Dis. 4, e623-7 (2013).

14. Kuchibhotla, K. V, Lattarulo, C. R., Hyman, B. T. \& Bacskai, B. J. Synchronous hyperactivity and intercellular calcium waves in astrocytes in Alzheimer mice. Science 323, 1211-5 (2009).

15. Talantova, M. et al. A $\beta$ induces astrocytic glutamate release, extrasynaptic NMDA receptor activation, and synaptic loss. Proc. Natl. Acad. Sci. U. S. A. 110, E2518-27 (2013).

16. Holtzclaw, L. A., Pandhit, S., Bare, D. J., Mignery, G. A. \& Russell, J. T. Astrocytes in adult rat brain express type 2 inositol 1,4,5-trisphosphate receptors. Glia 39, 69-84 (2002).

17. Nakahara, K., Okada, M. \& Nakanishi, S. The metabotropic glutamate receptor mGluR5 induces calcium oscillations in cultured astrocytes via protein kinase C phosphorylation. J. Neurochem. 69, 1467-75 (1997).

18. Van Den Pol, A. N., Romano, C. \& Ghosh, P. Metabotropic glutamate receptor mGluR5 subcellular distribution and developmental expression in hypothalamus. J. Comp. Neurol. 362, 134-150 (1995).

19. Bushong, E. a, Martone, M. E., Jones, Y. Z. \& Ellisman, M. H. Protoplasmic astrocytes in CA1 stratum radiatum occupy separate anatomical domains. J. Neurosci. 22, 183-192 (2002).

20. Halassa, M. M., Fellin, T. \& Haydon, P. G. The tripartite synapse: roles for gliotransmission in health and disease. Trends Mol. Med. 13, 54-63 (2007).

21. Arizono, M. et al. Receptor-selective diffusion barrier enhances sensitivity of astrocytic processes to metabotropic glutamate receptor stimulation. Sci. Signal. 5, (2012).

22. Marchaland, J. et al. Fast Subplasma Membrane Ca2+ Transients Control Exo-Endocytosis of Synaptic-Like Microvesicles in Astrocytes. J. Neurosci. 28, 9122-9132 (2008).

23. Shigetomi, E., Kracun, S., Sofroniew, M. V. \& Khakh, B. S. A genetically targeted optical sensor to monitor calcium signals in astrocyte processes. Nat. Neurosci. 13, 759-766 (2010).

24. Santello, M., Bezzi, P. \& Volterra, A. TNF $\alpha$ Controls Glutamatergic Gliotransmission in the Hippocampal Dentate Gyrus. Neuron 69, 988-1001 (2011).

25. Otsu, Y. et al. Calcium dynamics in astrocyte processes during neurovascular coupling. Nat. Neurosci. 18, 210-8 (2015).

26. Bezzi, P. et al. Prostaglandins stimulate calcium-dependent glutamate release in astrocytes. Nature 391, 281-285 (1998).

27. Bowser, D. N. \& Khakh, B. S. Two forms of single-vesicle astrocyte exocytosis imaged with total internal reflection fluorescence microscopy. Proc. Natl. Acad. Sci. U. S. A. 104, 4212-4217 (2007).

28. Mittelsteadt, T. et al. Differential mRNA expression patterns of the synaptotagmin gene family in the rodent brain. J. Comp. Neurol. 512, 514-528 (2009). 
29. Zhang, Q., Fukuda, M., Van Bockstaele, E., Pascual, O. \& Haydon, P. G. Synaptotagmin IV regulates glial glutamate release. Proc. Natl. Acad. Sci. 101, 9441-9446 (2004).

30. Bacaj, T. et al. Article Synaptotagmin-1 and Synaptotagmin-7 Trigger Synchronous and Asynchronous Phases of Neurotransmitter Release. 947-959 (2013).

31. Wang, C. T. et al. Different domains of synaptotagmin control the choice between kiss-and-run and full fusion. Nature 424, 943-947 (2003).

32. Malarkey, E. B. \& Parpura, V. Temporal characteristics of vesicular fusion in astrocytes: examination of synaptobrevin 2-laden vesicles at single vesicle resolution. J. Physiol. 589, 42714300 (2011).

33. Wolfes, A. C. et al. A novel method for culturing stellate astrocytes reveals spatially distinct Ca 2+ signaling and vesicle recycling in astrocytic processes. J. Gen. Physiol. 149, 1-22 (2017).

34. Angulo, M. C. Glutamate Released from Glial Cells Synchronizes Neuronal Activity in the Hippocampus. J. Neurosci. 24, 6920-6927 (2004).

35. Neves, G., Cooke, S. F. \& Bliss, T. V. P. Synaptic plasticity, memory and the hippocampus: a neural network approach to causality. Nat. Rev. Neurosci. (2008). doi:10.1038/nrn2303

36. Koch, C. \& Zador, A. The function of dendritic spines: devices subserving biochemical rather than electrical compartmentalization. J. Neurosci. (2018). doi:10.1523/jneurosci.13-02-00413.1993

37. Bourne, J. N. \& Harris, K. M. Balancing structure and function at hippocampal dendritic spines. Annu. Rev. Neurosci. (2008). doi:10.1146/annurev.neuro.31.060407.125646

38. Ventura, R. \& Harris, K. M. Three-dimensional relationships between hippocampal synapses and astrocytes. J. Neurosci. 19, 6897-6906 (1999).

39. Halassa, M. M., Fellin, T., Takano, H., Dong, J. \& Haydon, P. G. Synaptic Islands Defined by the Territory of a Single Astrocyte. J. Neurosci. 27, 6473-6477 (2007).

40. Honsek, S. D., Walz, C., Kafitz, K. W. \& Rose, C. R. Astrocyte calcium signals at Schaffer collateral to CA1 pyramidal cell synapses correlate with the number of activated synapses but not with synaptic strength. Hippocampus 22, 29-42 (2012).

41. Höfer, T., Venance, L. \& Giaume, C. Control and plasticity of intercellular calcium waves in astrocytes: a modeling approach. J. Neurosci. 22, 4850-4859 (2002).

42. Bellinger, S. Modeling calcium wave oscillations in astrocytes. Neurocomputing 65-66, 843-850 (2005).

43. Bennett, M. R., Farnell, L. \& Gibson, W. G. A Quantitative Model of Purinergic Junctional Transmission of Calcium Waves in Astrocyte Networks. Biophys. J. 89, 2235-2250 (2005).

44. Stamatakis, M. \& Mantzaris, N. V. Modeling of ATP-mediated signal transduction and wave propagation in astrocytic cellular networks. J. Theor. Biol. 241, 649-668 (2006). 
45. Perea, G. Properties of Synaptically Evoked Astrocyte Calcium Signal Reveal Synaptic Information Processing by Astrocytes. J. Neurosci. 25, 2192-2203 (2005).

46. Gaffney, M. PDFlib PLOP : PDF Linearization, Optimization, Protection Page inserted by evaluation version Keeping Land in Capital Theory. Am. J. Econ. Sociol. 67, 119-141 (2008).

47. Maravall, M., Mainen, Z. F., Sabatini, B. L. \& Svoboda, K. Estimating intracellular calcium concentrations and buffering without wavelength ratioing. Biophys. J. 78, 2655-2667 (2000).

48. Sabatini, B. L., Oertner, T. G. \& Svoboda, K. The Life Cycle of Ca2+ Ions in Dendritic Spines. Neuron 33, 439-452 (2002).

49. Burdakov, D., Petersen, O. H. \& Verkhratsky, A. Intraluminal calcium as a primary regulator of endoplasmic reticulum function. 38, 303-310 (2005).

50. De Young, G. W. \& Keizer, J. A single-pool inositol 1,4,5-trisphosphate-receptor-based model for agonist-stimulated oscillations in Ca2+ concentration. Proc. Natl. Acad. Sci. 89, 9895-9899 (1992).

51. Shuai, J.-W. \& Jung, P. Stochastic properties of $\mathrm{Ca}(2+)$ release of inositol 1,4,5-trisphosphate receptor clusters. Biophys. J. 83, 87-97 (2002).

52. Swillens, S., Dupont, G., Combettes, L. \& Champeil, P. From calcium blips to calcium puffs: theoretical analysis of the requirements for interchannel communication. Proc. Natl. Acad. Sci. U. S. A. 96, 13750-13755 (1999).

53. Camello, C., Lomax, R., Petersen, O. H. \& Tepikin, A. V. Calcium leak from intracellular store The enigma of calcium signalling. Cell Calcium 32, 355-361 (2002).

54. De Pittà, M., Goldberg, M., Volman, V., Berry, H. \& Ben-Jacob, E. Glutamate regulation of calcium and IP3 oscillating and pulsating dynamics in astrocytes. J. Biol. Phys. (2009). doi:10.1007/s10867-009-9155-y

55. Rao, S. K., Huynh, C., Proux-Gillardeaux, V., Galli, T. \& Andrews, N. W. Identification of SNAREs Involved in Synaptotagmin VII-regulated Lysosomal Exocytosis. J. Biol. Chem. 279, 20471-20479 (2004).

56. Crippa, D. et al. Synaptobrevin2-expressing vesicles in rat astrocytes: insights into molecular characterization, dynamics and exocytosis. J. Physiol. 570, 567-582 (2006).

57. Calì, C., Marchaland, J., Regazzi, R. \& Bezzi, P. SDF 1-alpha (CXCL12) triggers glutamate exocytosis from astrocytes on a millisecond time scale: Imaging analysis at the single-vesicle level with TIRF microscopy. J. Neuroimmunol. 198, 82-91 (2008).

58. Domercq, M. et al. P2Y1 receptor-evoked glutamate exocytosis from astrocytes: Control by tumor necrosis factor-?? and prostaglandins. J. Biol. Chem. 281, 30684-30696 (2006).

59. Oberheim, N. a et al. Uniquely hominid features of adult human astrocytes. J. Neurosci. 29, 32763287 (2009). 
60. Bergles, D. E. \& Jahr, C. E. Synaptic activation of glutamate transporters in hippocampal astrocytes. Neuron 19, 1297-1308 (1997).

61. Pinsky, P. F. \& Rinzel, J. Synchrony measures for biological neural networks. Biol. Cybern. 73, 129-137 (1995).

62. Strogatz, S. H. \& Mirollo, R. E. Stability of incoherence in a population of coupled oscillators. $J$. Stat. Phys. 63, 613-635 (1991).

63. Aertsen, a M., Gerstein, G. L., Habib, M. K. \& Palm, G. Dynamics of neuronal firing correlation: modulation of 'effective connectivity'. J. Neurophysiol. 61, 900-917 (1989).

64. Parpura, V. \& Zorec, R. Gliotransmission: Exocytotic release from astrocytes. Brain Res. Rev. 63, 83-92 (2010).

65. Hui, E. et al. Three distinct kinetic groupings of the synaptotagmin family: candidate sensors for rapid and delayed exocytosis. Proc. Natl. Acad. Sci. U. S. A. 102, 5210-4 (2005).

66. Kreft, M. et al. Properties of Ca2+-dependent exocytosis in cultured astrocytes. Glia 46, 437-445 (2004).

67. Südhof, T. C. the Synaptic Vesicle Cycle Neurotransmitter Release and the Synaptic Vesicle Cycle. Annu. Rev. Neurosci 27, 509-47 (2004).

68. Nadkarni, S., Bartol, T. M., Sejnowski, T. J. \& Levine, H. Modelling vesicular release at hippocampal synapses. PLoS Comput. Biol. 6, (2010).

69. Sun, J. et al. A dual-Ca2+-sensor model for neurotransmitter release in a central synapse. Nature 450, 676-682 (2007).

70. Casley, C. S. et al. Up-regulation of astrocyte metabotropic glutamate receptor 5 by amyloid- $\beta$ peptide. Brain Res. 1260, 65-75 (2009).

71. Lim, D. et al. Amyloid beta deregulates astroglial mGluR5-mediated calcium signaling via calcineurin and Nf-kB. Glia 61, 1134-1145 (2013).

72. Berrocal, M. et al. Altered Ca2+ dependence of synaptosomal plasma membrane Ca2+-ATPase in human brain affected by Alzheimer's disease. FASEB J. 23, 1826-1834 (2009).

73. Mata, A. M., Berrocal, M. \& Sepúlveda, M. R. Impairment of the activity of the plasma membrane $\mathrm{Ca}^{2+}$-ATPase in Alzheimer's disease. Biochem. Soc. Trans. 39, 819-822 (2011).

74. Berrocal, M., Sepulveda, M. R., Vazquez-Hernandez, M. \& Mata, A. M. Calmodulin antagonizes amyloid- $\beta$ peptides-mediated inhibition of brain plasma membrane Ca2+-ATPase. Biochim. Biophys. Acta - Mol. Basis Dis. 1822, 961-969 (2012).

75. Bernstein, M., Behnisch, T., Balschun, D., Reymann, K. G. \& Reiser, G. Pharmacological characterisation of metabotropic glutamatergic and purinergic receptors linked to $\mathrm{Ca} 2+$ signalling in hippocampal astrocytes. Neuropharmacology 37, 169-178 (1998). 
76. Pasti, L., Volterra, A., Pozzan, T. \& Carmignoto, G. Intracellular calcium oscillations in astrocytes: a highly plastic, bidirectional form of communication between neurons and astrocytes in situ. J. Neurosci. 17, 7817-30 (1997).

77. Chow, S.-K., Yu, D., MacDonald, C. L., Buibas, M. \& Silva, G. A. Amyloid $\beta$-Peptide Directly Induces Spontaneous Calcium Transients, Delayed Intercellular Calcium Waves and Gliosis in Rat Cortical Astrocytes. ASN Neuro 2, AN20090035 (2010).

78. Takano, T., Han, X., Deane, R., Zlokovic, B. \& Nedergaard, M. Two-photon imaging of astrocytic $\mathrm{Ca} 2+$ signaling and the microvasculature in experimental mice models of Alzheimer's disease. Ann. N. Y. Acad. Sci. 1097, 40-50 (2007).

79. Szabó, Z. et al. Extensive astrocyte synchronization advances neuronal coupling in slow wave activity in vivo. Sci. Rep. 7, 1-18 (2017).

80. Sasaki, T. et al. Astrocyte calcium signalling orchestrates neuronal synchronization in organotypic hippocampal slices. J. Physiol. 592, 2771-83 (2014).

81. Kékesi, O., Ioja, E., Szabó, Z., Kardos, J. \& Héja, L. Recurrent seizure-like events are associated with coupled astroglial synchronization. Front. Cell. Neurosci. 9, 215 (2015).

82. Selkoe, D. J. Alzheimer's disease is a synaptic failure. Science (2002). doi:10.1126/science.1074069

83. Kang, M. \& Othmer, H. G. Spatiotemporal characteristics of calcium dynamics in astrocytes. Chaos 19, (2009).

84. Lavrentovich, M. \& Ã, S. H. A mathematical model of spontaneous calcium ( II ) oscillations in astrocytes. 251, 553-560 (2008).

85. Araque, A. et al. Gliotransmitters travel in time and space. Neuron 81, 728-739 (2014).

86. Covelo, A. \& Araque, A. Neuronal activity determines distinct gliotransmitter release from a single astrocyte. Elife (2018). doi:10.7554/elife.32237

87. Di Castro, M. A. et al. Local $\mathrm{Ca} 2+$ detection and modulation of synaptic release by astrocytes. Nat. Neurosci. 14, 1276-1284 (2011).

88. Liu, T. et al. Calcium triggers exocytosis from two types of organelles in a single astrocyte. $J$. Neurosci. 31, 10593-10601 (2011).

89. Nadkarni, S., Jung, P. \& Levine, H. Astrocytes optimize the synaptic transmission of information. PLoS Comput. Biol. 4, (2008).

90. Pivneva, T. et al. Store-operated Ca2+ entry in astrocytes: Different spatial arrangement of endoplasmic reticulum explains functional diversity in vitro and in situ. Cell Calcium 43, 591-601 (2008).

91. Asada, A. et al. Subtle modulation of ongoing calcium dynamics in astrocytic microdomains by sensory inputs. Physiol. Rep. 3, e12454 (2015). 
92. Skupin, A., Kettenmann, H. \& Falcke, M. Calcium signals driven by single channel noise. PLoS Comput. Biol. 6, 17-19 (2010).

93. Balázs, R. et al. Metabotropic Glutamate Receptor mGluR5 in Astrocytes: Pharmacological Properties and Agonist Regulation. J. Neurochem. 69, 151-163 (2002).

94. Malli, R., Frieden, M., Hunkova, M., Trenker, M. \& Graier, W. F. Ca2+ refilling of the endoplasmic reticulum is largely preserved albeit reduced Ca2+ entry in endothelial cells. Cell Calcium 41, 63-76 (2007).

95. Samtleben, S. et al. Direct Imaging of ER Calcium with Targeted-Esterase Induced Dye Loading (TED). J. Vis. Exp. 1-17 (2013). doi:10.3791/50317

96. Solovyova, N. Ca2+ dynamics in the lumen of the endoplasmic reticulum in sensory neurons: direct visualization of $\mathrm{Ca} 2+$-induced $\mathrm{Ca} 2+$ release triggered by physiological $\mathrm{Ca} 2+$ entry. $E M B O$ J. 21, 622-630 (2002).

97. Zhang, Z. et al. Regulated ATP release from astrocytes through lysosome exocytosis. Nat. Cell Biol. 9, 945-953 (2007).

98. Lee, L., Kosuri, P. \& Arancio, O. Picomolar amyloid- $\beta$ peptides enhance spontaneous astrocyte calcium transients. J. Alzheimer's Dis. 38, 49-62 (2014).

99. Fellin, T. et al. Neuronal synchrony mediated by astrocytic glutamate through activation of extrasynaptic NMDA receptors. Neuron 43, 729-743 (2004).

100. Bartol, T. M., Keller, D. X., Kinney, J. P. \& Bajaj, C. L. Computational reconstitution of spine calcium transients from individual proteins. 7, 1-24 (2015).

101. Swaminathan, D., Ullah, G. \& Jung, P. A simple sequential-binding model for calcium puffs. Chaos 19, (2009).

102. Higgins, E. R., Cannell, M. B. \& Sneyd, J. A buffering SERCA pump in models of calcium dynamics. Biophys. J. 91, 151-163 (2006).

103. Ullah, G., Jung, P. \& Cornell-Bell, a. H. Anti-phase calcium oscillations in astrocytes via inositol (1, 4, 5)-trisphosphate regeneration. Cell Calcium 39, 197-208 (2006).

104. Østby, I. et al. Astrocytic mechanisms explaining neural-activity-induced shrinkage of extraneuronal space. PLoS Comput. Biol. 5, (2009).

105. Grosche, A. et al. Versatile and Simple Approach to Determine Astrocyte Territories in Mouse Neocortex and Hippocampus. PLoS One 8, (2013).

106. Li, Y. X. \& Rinzel, J. Equations for InsP3receptor-mediated [Ca2+](i) oscillations derived from a detailed kinetic model: A hodgkin-huxley like formalism. Journal of Theoretical Biology 166, 461-473 (1994).

107. Riera, J., Hatanaka, R., Uchida, T., Ozaki, T. \& Kawashima, R. Quantifying the uncertainty of spontaneous $\mathrm{Ca} 2+$ oscillations in astrocytes: Particulars of Alzheimer's disease. Biophys. J. 101, 554-564 (2011). 
108. Wade, J. J., McDaid, L. J., Harkin, J., Crunelli, V. \& Kelso, J. a S. Bidirectional coupling between astrocytes and neurons mediates learning and dynamic coordination in the brain: A multiple modeling approach. PLoS One 6, 1-24 (2011).

\section{Acknowledgements}

AGP was supported by a Postdoctoral Fellowship from Indian Institute of Science Education and Research Pune (IISER-P/Ext/PDRF/AP-20145047/10/2016) and Wellcome Trust/DBT India. S.N. was funded b y Wellcome Trust/DBT India Alliance (IA/I/12/1/500529) and Indian Institute of Science Education and Research Pune.

\section{Author Contributions}

A.G.P. and S.N. designed the research and wrote the paper. A.G.P. wrote the codes to build the model, perform the simulations and analyzed the data.

Competing interests: The authors declare no competing financial interests

\section{Figures}

Figure 1. Description of the $\mathrm{Ca}^{2+}$ model and characterization of mGluR-mediated $\mathrm{Ca}^{2+}$ events. a Schematic of the single astrocyte process model with all the molecular components for $\mathrm{Ca}^{2+}$ signaling. $\mathbf{b}$ Representative $\mathrm{Ca}^{2+}$ responses from a set of independent trials of an astrocytic process stimulated with DHPG $(100 \mu \mathrm{M}, 2 \mathrm{sec})$. $\mathbf{c}$ Overlay of individual $\mathrm{Ca}^{2+}$ events aligned to the peak. d Peak amplitude histogram of $\mathrm{Ca}^{2+}$ events from a set of 400 independent trials that reproduced experimental data (f). e Distribution of $\mathrm{Ca}^{2+}$ event rise times. $\mathbf{f}$ Comparison of mGluR-mediated $\mathrm{Ca}^{2+}$ event histograms from the model and experimental data (Marchaland et. $\mathrm{al}^{22}$ ). Inset Cumulative distribution of $\mathrm{Ca}^{2+}$ events from the 
model and experiment, indicating that the model captures more than $90 \%$ of the all events. Gray lines indicate Gaussian fits.

Figure 2. The gliotransmsitter release model and characterization of mGluR-mediated release events. a Schematic of the gliotransmitter release model, indicating the two synaptotagmins (Syt4 \& Syt7) that independently trigger kiss-and-run and full fusion releases from docked (resident) and mobile vesicles, respectively. b Total gliotransmitter release rate from an astrocytic process whose intracellular $\mathrm{Ca}^{2+}$ is clamped at different concentration steps. $\mathbf{c} \mathrm{Ca}^{2+}$ dependence of peak release rate is fitted with a Hill equation. d Time of peak release rate decrease exponentially with increasing cytosolic $\mathrm{Ca}^{2+}$ levels. e Perievent raster plot of mGluR-mediated kiss-and-run and full fusion events from a subset of simulation trials. f Comparison of temporal histogram of mGluR-mediated release events collected from 400 independent simulation trials and experimental data (Marchaland et al. ${ }^{22}$ ). Inset Cumulative histogram of gliotransmitter release from the single process model versus experimental data (Marchaland et al. ${ }^{22}$ ). $\mathbf{g}$ Distribution of kiss-and-run and full fusion releases from 400 independent trials of a single astrocyte process stimulated with DHPG $(100 \mu \mathrm{M}, 2 \mathrm{sec}) . \mathbf{h}$ Kinetics of release, endocytosis, and reacidification of docked vesicles after stimulation with DHPG. i Kinetics of release, endocytosis, and reacidification of mobile vesicles.

Figure 3. A D related alterations in mGluR and PMCA signaling modifies $\mathrm{Ca}^{2+}$ activity of astrocytic process at resting state and when stimulated by glutamate. a Dose response curve for mGluR-dependent $\mathrm{IP}_{3}$ production is shifted leftward in the AD-mGluR astrocytic process. $\mathbf{b}$ Intracellular $\mathrm{Ca}^{2+}$ dependence of PMCA steady state $\mathrm{Ca}^{2+}$ flux is shifted to the right in the AD-PMCA astrocytic process. $\mathbf{c}$ Resting cytosolic $\mathrm{Ca}^{2+}$ level is higher in astrocytic process with impaired PMCA activity. $\mathbf{d}$ Resting $\mathrm{IP}_{3}$ levels are not affected by AD-related molecular signaling. e Resting ER $\mathrm{Ca}^{2+}$ level is enhanced in $\mathrm{AD}$ astrocytic processes with altered PMCA signaling. f-i Representative $\mathrm{Ca}^{2+}$ responses from control and $\mathrm{AD}$ astrocytic 
processes that are stimulated with a prolonged glutamate pulse $(6 \mu \mathrm{M}, 120 \mathrm{~s})$. j Glutamate-induced $\mathrm{Ca}^{2+}$ responsiveness is enhanced in all $\mathrm{AD}$ astrocytic processes. $\mathbf{k} \mathrm{Ca}^{2+}$ event rate during glutamate stimulation is higher in all $\mathrm{AD}$ astrocyte groups when compared to the control group. Solid lines are Hill equation fits and vertical dotted lines indicate glutamate concentrations at half-maximal response.

Figure 4. Spontaneous and evoked $\mathrm{Ca}^{2+}$ activity are increased in $\mathrm{AD}$ astrocytic processes with $\mathrm{AD}$ pathology. a-d $\mathrm{Ca}^{2+}$ transients recorded from control and $\mathrm{AD}$ astrocytic processes stimulated with spillover glutamate from presynaptic release at $3 \mathrm{~Hz}$. Dotted boxes indicate the part where astrocytic process display spontaneous $\mathrm{Ca}^{2+}$ transients. e The rate of $\mathrm{Ca}^{2+}$ event rate increased non-linearly with stimulation frequency. Solid lines are fits to a Hill equation and dotted vertical lines indicate stimulation frequency for half-maximal response. Risetime (f), full-width at half-maximum (g) and decay time (h) of $\mathrm{Ca}^{2+}$ events averaged for all the events across 1000 independent simulation trials for different frequencies over the entire stimulation regime are lower in AD groups with aberrant PMCA, but not mGluR, signaling.

Figure 5. A $\beta$-induced alteration in PMCA signaling favors full fusion release over kiss-and-run exocytosis. a-d Kiss-and-run (red) and full fusion (blue) release events recorded from control and AD astrocytic processes stimulated with spillover glutamate from neuronal release at $3 \mathrm{~Hz}$. e A $\beta$-induced alterations in PMCA signaling abolished the logistic relationship between kiss-and-run release and glutamate stimulation rate. $\mathbf{f}$ Reduced PMCA activity in AD processes lead to a leftward shift in full fusion release rate. $\mathbf{g}$ The half maximum of total release rate is lowered in all astrocytic processes with AD pathology. Dotted lines indicate the frequency of half-maximal response for each group.

Figure 6. Synchrony (phase coherence) of $\mathrm{Ca}^{2+}$ events, but not release events, is enhanced in astrocytic processes with AD-related molecular alterations. a Polar histogram of phases of $\mathrm{Ca}^{2+}$ event from 1000 
independent simulation trials of astrocytic processes from control and AD groups that are stimulated with neuronal glutamate release at $0.2 \mathrm{~Hz}$. $\mathbf{~} \mathrm{Ca}^{2+}$ synchrony is high in the AD-mGluR when stimulated at low frequencies. The arrow indicates synchrony for $0.2 \mathrm{~Hz}$ stimulation. c Polarphase histogram of gliotransmitter release events from 1000 independent astrocytic processes from control and AD groups stimulated with neuronal glutamate release at $0.2 \mathrm{~Hz}$. d Synchrony of release events are not much different for the AD groups when compared to control.

Figure 7. Temporal relationship between $\mathrm{Ca}^{2+}$ and release events is reduced in astrocytic processes with AD pathology. a nJPSTH (left), coincidence (top) and cross correlation (bottom) histograms between $\mathrm{Ca}^{2+}$ and release events computed from 1000 independent simulation trials of the control astrocyte process stimulated with neuronal glutamate release at $10 \mathrm{~Hz}$. At the bottom and left side of the nJPSTH are PSTHs for $\mathrm{Ca}^{2+}$ and gliotransmitter release events. b-d nJPSTH, coincidence and cross correlation time histograms of $\mathrm{AD}-\mathrm{mGluR}, \mathrm{AD}-\mathrm{PMCA}$ and $\mathrm{AD}-(\mathrm{mGluR}+\mathrm{PMCA})$ groups. e Peak cross correlations between $\mathrm{Ca}^{2+}$ and release events at different stimulation rates are low in all $\mathrm{AD}$ astrocytic processes. $\mathbf{f}$ The average number of docked vesicles computed from 1000 independent trials is low in all AD groups. $\mathbf{g}$ The linear relationship between peak cross correlation and docked vesicles at the theta stimulation range (2-10 $\mathrm{Hz}$ ) indicates rapid vesicle depletion in $\mathrm{AD}$ processes as a mechanism for the loss in temporal association between $\mathrm{Ca}^{2+}$ and release events. Fits to Hill equations are in solid lines.

\section{Tables}

Table 1. $\mathrm{Ca}^{2+}$ fluxes in the model 


\begin{tabular}{|c|c|}
\hline Component name & Equation \\
\hline $\mathrm{PMCA}^{68}$ & $\begin{array}{l}J_{P M C A}=\left(-M_{0} C a_{c y t}^{2+} k_{f 1}\right)+\left(M_{1} k_{b 1}\right)+\left(M_{0} k_{l}\right) \\
\frac{d}{d t} M_{0}=\left(-M_{0} C a_{c y t}^{2+} k_{f 1}\right)+\left(M_{1} k_{b 1}\right)+\left(M_{2} k_{f 3}\right) \\
\frac{d}{d t} M_{1}=\left(-M_{1} k_{b 1}\right)+\left(M_{0} C a_{c y t}^{2+} k_{f 1}\right)+\left(-M_{1} k_{f 2}\right) \\
\frac{d}{d t} M_{2}=\left(-M_{2} k_{f 3}\right)+\left(M_{1} k_{f 2}\right)\end{array}$ \\
\hline Cytosol $\mathrm{Ca}^{2+}$ buffer $^{100}$ & $\begin{array}{l}J_{C y t B}=-C a_{c y t}^{2+} C_{0} k_{f}+C_{1} k_{b} \\
\frac{d}{d t} C_{0}=\left(-C_{0} C a_{c y t}^{2+} k_{f}\right)+\left(C_{1} k_{b}\right) \\
\frac{d}{d t} C_{1}=\left(-C_{1} k_{b}\right)+\left(C_{0} C a_{c y t}^{2+} k_{f}\right)\end{array}$ \\
\hline SERCA $^{101}$ & $J_{S E R C A}=V_{\max } \frac{\left(C a_{c y t}^{2+}\right)^{2}}{\left(C a_{c y t}^{2+}\right)^{2}+\left(k_{a}\right)^{2}}$ \\
\hline ER-leak $^{50}$ & $J_{\text {ER-leak }}=k_{\text {leak }}\left(C a_{E R}^{2+}-C a_{c y t}^{2+}\right)$ \\
\hline $\mathrm{IP}_{3}$ receptor $^{51}$ & $\begin{array}{l}J_{I P 3 R}=v_{\max } m_{\infty}^{3} n_{\infty}^{3} h^{3}\left(C a_{E R}^{2+}-C a_{c y t}^{2+}\right) \\
\frac{d}{d t} h=\alpha_{h}(1-h)-\beta_{h} h+\zeta(t) \\
\langle\zeta(t)\rangle=0 \\
\left\langle\zeta(t) \zeta\left(t^{\prime}\right)\right\rangle=\frac{\left(\alpha_{h}(1-h)+\beta_{h} h\right)}{n} \delta\left(t-t^{\prime}\right) \\
d_{i}=\frac{b_{i}}{a_{i}}, \quad q_{2}=d_{2}\left(\frac{I P_{3}+d_{1}}{I P_{3}+d_{3}}\right) \\
n_{\infty}=\frac{I P_{3}}{I P_{3}+d_{1}}, \quad m_{\infty}=\frac{C a_{c y t}^{2+}}{C a_{c y t}^{2+}+d_{5}} \\
\alpha_{h}=a_{2} d_{2} \frac{\left(I P_{3}+d_{1}\right)}{\left(I P_{3}+d_{3}\right)}, \quad \beta_{h}=a_{2} C a_{c y t}^{2+}\end{array}$ \\
\hline $\mathrm{ER} \mathrm{Ca}^{2+}$ buffer $^{102}$ & $\begin{array}{l}J_{E R B}=-C a_{E R}^{2+} B_{0} k_{f}+B_{1} k_{b} \\
\frac{d}{d t} B_{0}=\left(-B_{0} C a_{E R}^{2+} k_{f}\right)+\left(B_{1} k_{b}\right) \\
\frac{d}{d t} B_{1}=\left(-B_{1} k_{b}\right)+\left(B_{0} C a_{E R}^{2+} k_{f}\right)\end{array}$ \\
\hline $\begin{array}{l}\text { Synaptotagmin } 4 \& \\
7^{68,69}\end{array}$ & $\begin{aligned} J_{S y t}= & J_{S y t 4}+J_{S y t 7} \\
J_{S y t 4}= & \left(-2 S_{0} k_{f 4} C a_{c y t}^{2+}\right)+\left(S_{1} k_{b 4}\right)+\left(-S_{1} k_{f 4} C a_{c y t}^{2+}\right)+\left(2 S_{2} k_{b 4} b_{4}\right) \\
J_{S y t 7}= & +\left(-5 Y_{0} k_{f 7} C a_{c y t}^{2+}\right)+\left(Y_{1} k_{b 7}\right)+\left(-4 Y_{1} k_{f 7} C a_{c y t}^{2+}\right)+\left(2 Y_{2} k_{b 7} b_{7}\right) \\
& +\left(-3 Y_{2} k_{f 7} C a_{c y t}^{2+}\right)+\left(3 Y_{3} k_{b 7} b_{7}^{2}\right)+\left(-2 Y_{3} k_{f 7} C a_{c y t}^{2+}\right)+\left(4 Y_{4} k_{b 7} b_{7}^{3}\right) \\
& +\left(-Y_{4} k_{f 7} C a_{c y t}^{2+}\right)+\left(5 Y_{5} k_{b 7} b_{7}^{4}\right)\end{aligned}$ \\
\hline
\end{tabular}


Table 2. $\mathrm{IP}_{3}$ fluxes in the model

\begin{tabular}{|l|l|}
\hline Component name & Equation \\
\hline $\mathrm{mGluR}^{103}$ & $J_{m G l u R}=V_{\max } \frac{(G l u)^{2}}{(G l u)^{2}+\left(k_{a}\right)^{2}}$ \\
\hline $\mathrm{IP}_{3}$ 3-kinase & \\
\hline $\mathrm{IP}_{3}$ 5-phosphatase & $J_{I P 3 K}=V_{\max } \frac{\left(C a_{c y t}^{2+}\right)^{4}}{\left(C a_{c y t}^{2+}\right)^{4}+\left(k_{a 1}\right)^{4}} \times \frac{I P_{3}}{I P_{3}+k_{a 2}}$ \\
\hline $\mathrm{PLC}_{\delta}^{44}$ & $J_{I P 3 P}=V_{\text {max }}\left(I P_{3}-I P_{3}^{\text {base }}\right)$ \\
\hline
\end{tabular}

Table 3. Model parameters for $\mathrm{Ca}^{2+}$ and $\mathrm{IP}_{3}$ dynamics

\begin{tabular}{|c|c|}
\hline Component name & Parameters \\
\hline $\mathrm{PMCA}^{68}$ & $\begin{array}{l}k_{f l}=15 \mu \mathrm{M}^{-1} \mathrm{~s}^{-1}, k_{b l}=20 \mathrm{~s}^{-1}, k_{f 2}=20 \mathrm{~s}^{-1}, k_{f 3}=100 \mathrm{~s}^{-1}, k_{l}=0.6 \mathrm{~s}^{-1}, \text { PMCA density }= \\
400 \mu \mathrm{m}^{-2}\end{array}$ \\
\hline Cytosol $\mathrm{Ca}^{2+}$ buffer ${ }^{100}$ & $\begin{array}{l}k_{f}=60 \mu \mathrm{M}^{-1} \mathrm{~s}^{-1}, k_{b}=1200 \mathrm{~s}^{-1}, \\
\text { buffer concentration }=50 \mu \mathrm{M}\end{array}$ \\
\hline SERCA $^{101}$ & $V_{\max }=250 \mu \mathrm{Ms}^{-1}, k_{a}=100 \mathrm{nM}, n=2$ \\
\hline ER-leak & $k_{l}=0.2 \mathrm{~s}^{-1}$ \\
\hline $\mathrm{IP}_{3}$ receptor $^{51}$ & $\begin{array}{l}\mathrm{a}_{1}=400 \mu \mathrm{Ms}^{-1}, \mathrm{a}_{2}=0.2 \mu \mathrm{M}^{-1} \mathrm{~s}^{-1}, \mathrm{a}_{3}=400 \mu \mathrm{M}^{-1} \mathrm{~s}^{-1}, \mathrm{a}_{4}=0.2 \mu \mathrm{M}^{-1} \mathrm{~s}^{-1}, \mathrm{a}_{5}=20 \mu \mathrm{M}^{-1} \mathrm{~s}^{-1}, \\
\mathrm{~b}_{1}=52 \mathrm{~s}^{-1}, \mathrm{~b}_{2}=0.2 \mathrm{~s}^{-1}, \mathrm{~b}_{3}=377.36 \mathrm{~s}^{-1}, \mathrm{~b}_{4}=0.02 \mathrm{~s}^{-1}, \mathrm{~b}_{5}=1.64 \mathrm{~s}^{-1}, v_{\max }=6.0 \mathrm{~s}^{-1}, \mathrm{n}= \\
5\end{array}$ \\
\hline $\mathrm{ER} \mathrm{Ca}^{2+}$ buffer ${ }^{102}$ & $k f 1=1 \mu \mathrm{M}^{-1} \mathrm{~s}^{-1}, k b 1=80 \mathrm{~s}^{-1}$, buffer concentration $=10 \mu \mathrm{M}$ \\
\hline mGluR $^{103}$ & $V_{\max }=0.65 \mu \mathrm{Ms}^{-1}, k_{a}=6 \mu \mathrm{M}, n=2$ \\
\hline $\mathrm{IP}_{3}$ 3-kinase ${ }^{54}$ & $V_{\max }=5 \mu \mathrm{Ms}^{-1}, k_{a l}=0.4 \mu \mathrm{M}, n_{1}=4, k_{a 2}=10 \mu \mathrm{M}, n_{2}=1$ \\
\hline $\mathrm{IP}_{3}$ 5-phosphatase $\mathrm{p}^{44,103}$ & $V_{\max }=1.25 \mathrm{~s}^{-1}$ \\
\hline $\mathrm{PLC}_{\delta}^{44}$ & $V_{\max }=0.02 \mu \mathrm{Ms}^{-1}, k_{a l}=1 \mu \mathrm{M}, n_{1}=2, k_{a 2}=1.5 \mu \mathrm{M}$ \\
\hline
\end{tabular}

Table 4. Model parameters for vesicular release (Adapted from multiple studies ${ }^{22,66,68,69}$ )

\begin{tabular}{|l|l|}
\hline Parameter name & Value \\
\hline Fraction of docked vesicles $\left(N_{d o c}\right)$ & 0.8 \\
\hline Fraction of mobile vesicles $\left(N_{m o b}\right)$ & 0.25 \\
\hline Syt4 association rate constant $\left(k_{f 4}\right)$ & $153 \mu \mathrm{M}^{-1} \mathrm{~s}^{-1}$ \\
\hline Syt4 dissociation rate constant $\left(k_{b 4}\right)$ & $3500 \mathrm{~s}^{-1}$ \\
\hline Syt4 cooperativity factor $\left(b_{4}\right)$ & 0.25 \\
\hline Syt4 spontaneous release rate $\left(r_{04}\right)$ & $0.000417 \mathrm{~s}^{-1}$ \\
\hline
\end{tabular}




\begin{tabular}{|l|l|}
\hline Syt $4 \mathrm{Ca}^{2+}$-evoked release rate $\left(r_{c a 4}\right)$ & $115 \mathrm{~s}^{-1}$ \\
\hline Fast release endocytosis rate $\left(k_{e f}\right)$ & $0.66 \mathrm{~s}^{-1}$ \\
\hline Fast release reacidification rate $\left(k_{a f}\right)$ & $0.6 \mathrm{~s}^{-1}$ \\
\hline Syt7 association rate constant $\left(k_{f 7}\right)$ & $3.82 \mu \mathrm{M}^{-1} \mathrm{~s}^{-1}$ \\
\hline Syt7 dissociation rate constant $\left(k_{b 7}\right)$ & $60 \mathrm{~s}^{-1}$ \\
\hline Syt7 cooperativity factor $\left(b_{7}\right)$ & 0.25 \\
\hline Syt7 spontaneous release rate $\left(r_{o 7}\right)$ & $0.000417 \mathrm{~s}^{-1}$ \\
\hline Syt7 $\mathrm{Ca}^{2+}$-evoked release rate $\left(r_{c a}\right)$ & $8 \mathrm{~s}^{-1}$ \\
\hline Slow release endocytosis rate $\left(k_{e s}\right)$ & $0.16 \mathrm{~s}^{-1}$ \\
\hline Slow release reacidification rate $\left(k_{a s}\right)$ & $0.052 \mathrm{~s}^{-1}$ \\
\hline Mobile vesicle replenishment rate & $0.615 \mathrm{~s}^{-1}$ \\
\hline Vesicle docking rate $\left(k_{d o c}\right)$ & $0.75 \mathrm{~s}^{-1}$ \\
\hline
\end{tabular}

Table 5. Additional parameters/details of the model

\begin{tabular}{|c|c|c|}
\hline Component name & Parameters & Notes/Reference \\
\hline Resting cytosol $\mathrm{Ca}^{2+}$ & $\sim 80 \mathrm{nM}$ & As reported by Kuchibhotla et al. ${ }^{14}$ \\
\hline Resting ER $\mathrm{Ca}^{2+}$ & $\sim 400 \mu \mathrm{M}$ & As reported by Burdakov et al. ${ }^{49}$ \\
\hline Resting $\mathrm{IP}_{3}$ & $160 \mathrm{nM}$ & Taken from Wade et al. ${ }^{108}$ wade 2011 \\
\hline External $\mathrm{Ca}^{2+}$ & $2 \mathrm{mM}$ & \\
\hline Cytosol volume $22,104,105$ & $0.37 \mu \mathrm{m}^{3}$ & \\
\hline Cytosol area $22,104,105$ & $4.92 \mu \mathrm{m}^{2}$ & \\
\hline ER volume & $0.185 \mu \mathrm{m}^{3}$ & $\begin{array}{l}\text { High ER to cytosol volume ratio in astrocytic process, } \\
\text { as suggested by Marchaland et al. }{ }^{22}\end{array}$ \\
\hline ER area $^{106,107}$ & $49.2 \mu \mathrm{m}^{2}$ & \\
\hline Specific capacitance & $1.0 \mu \mathrm{F} \mathrm{cm}^{-2}$ & \\
\hline
\end{tabular}


a

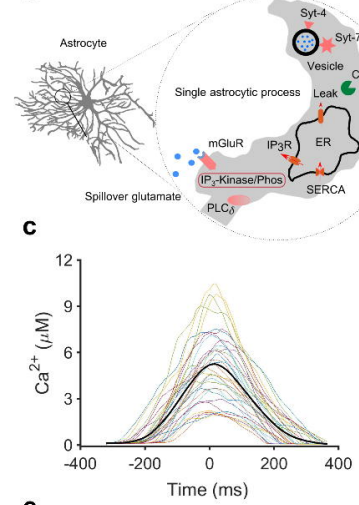

e

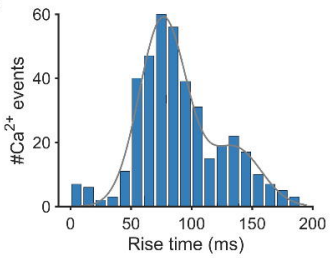

b
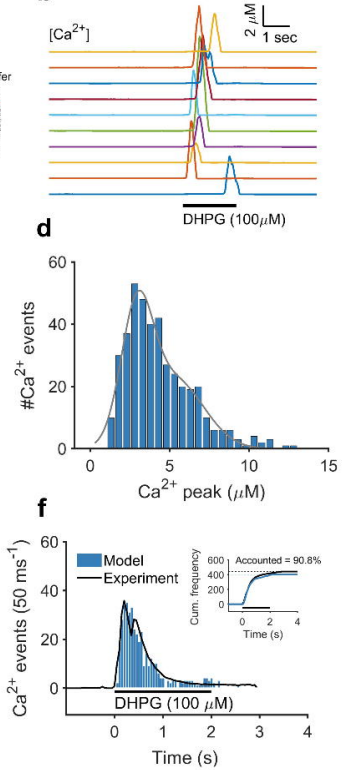
a

Fast release pathway

b

Slow release pathway
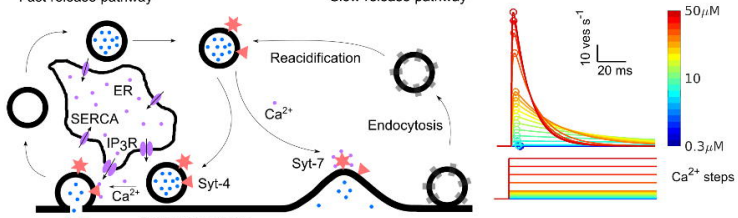

KR release

Resident vesicles

C FF release

.

d

e

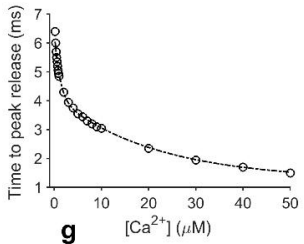

- KR release

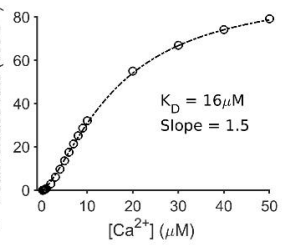

.

है

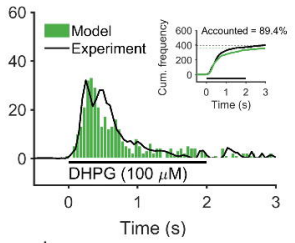

h
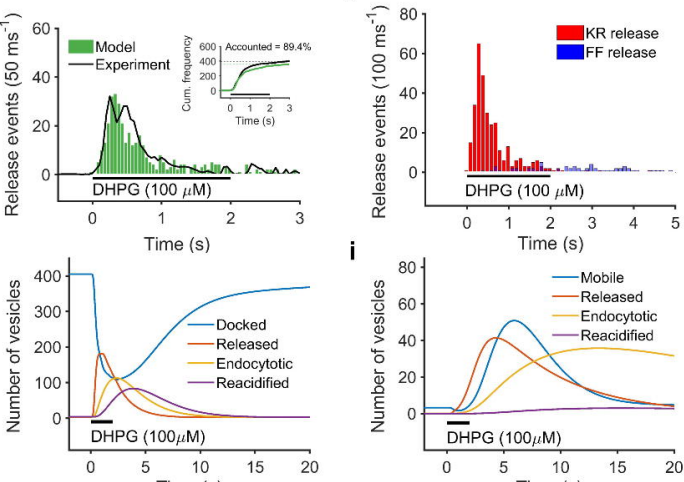

Time (s)

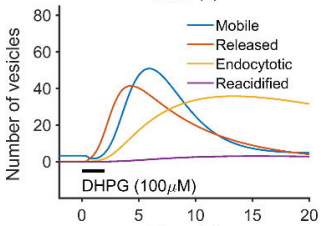

Time (s) 


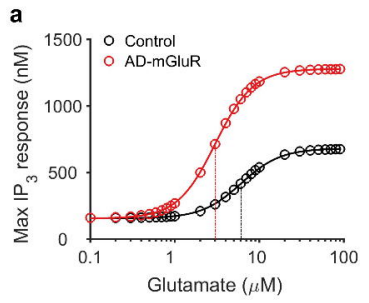

c

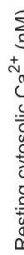

d b
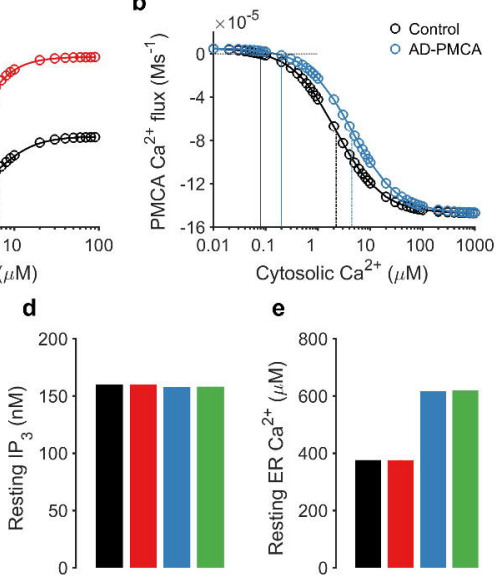

g

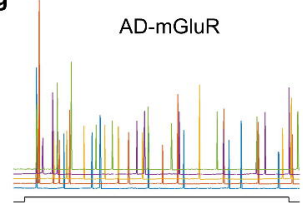

i

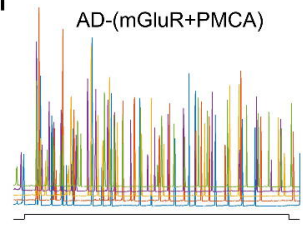

k




a

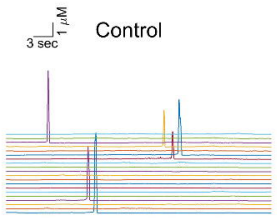

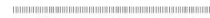
Glutamate release at $3 \mathrm{~Hz}$
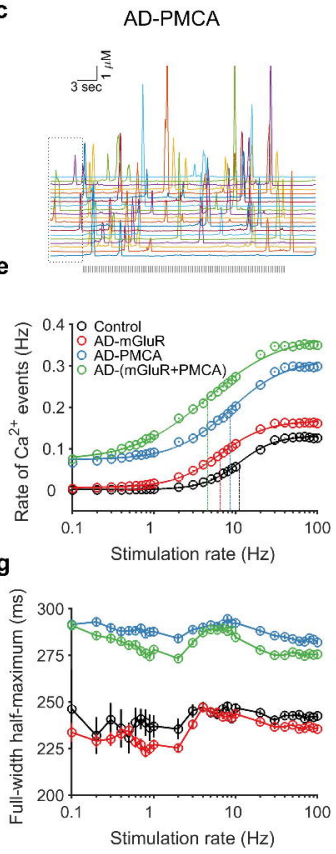

b
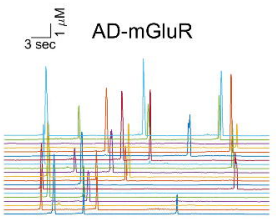

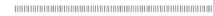

d
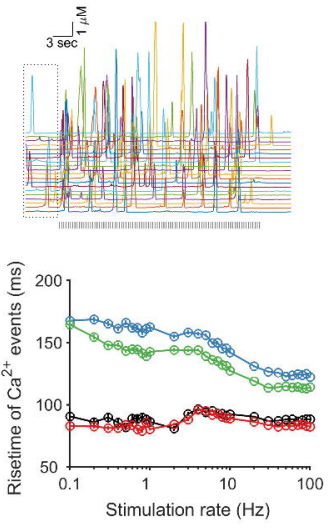

h

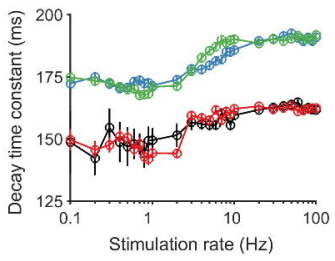


a

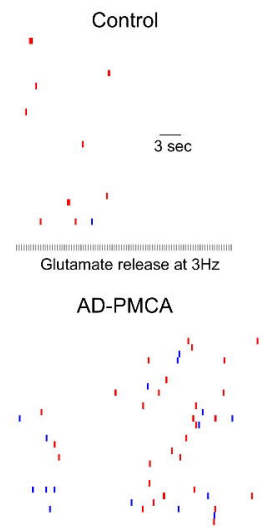

C

c

e

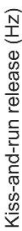

- Control

- AD-mGluR

- AD-PMCA

AD-(mGluR+PMCA)

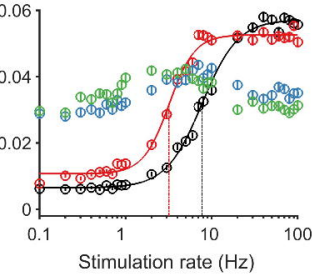

f

d

AD-mGluR

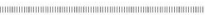

AD-(mGluR+PMCA)

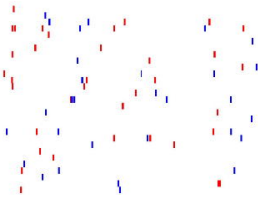

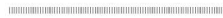

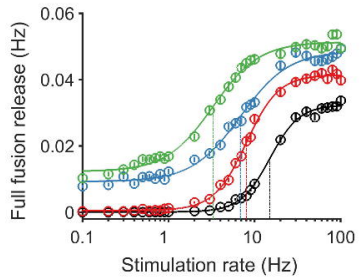

g

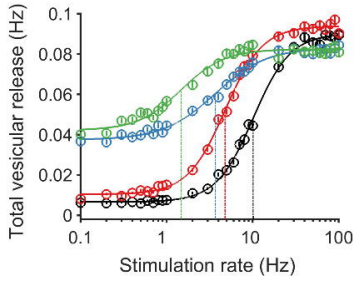


a Phase coherence histogram: calcium events $(0.2 \mathrm{~Hz})$

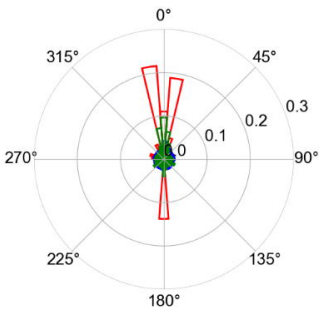

Phase coherence histogram:

release events $(0.2 \mathrm{~Hz})$

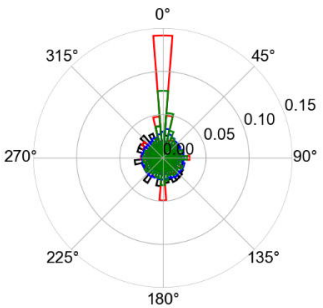

b

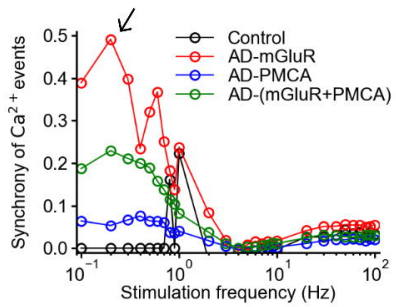

d 
